\title{
Analytical solution to the Riemann problem of three-phase flow in porous media
}

\author{
Ruben Juanes \\ Tadeusz W. Patzek \\ Department of Civil and Environmental Engineering \\ University of California at Berkeley \\ 631 Davis Hall, Berkeley, CA 94720 \\ Earth Sciences Division \\ Lawrence Berkeley National Lab \\ 1 Cyclotron Road Mailstop 90-1116, Berkeley, CA 94720 \\ Submitted to Transport in Porous Media
}

September 25, 2002

\begin{abstract}
In this paper we study one-dimensional three-phase flow of immiscible, incompressible fluids through porous media. The model uses the common multiphase flow extension of Darcy's equation, and does not include gravity and capillarity effects. Under these conditions, the mathematical problem reduces to a $2 \times 2$ system of conservation laws, whose essential features are: (1) the system is strictly hyperbolic; (2) both characteristic fields are nongenuinely nonlinear, with single, connected inflection loci. We argue that these are necessary properties for the solution to be physically sensible, and show they are natural extensions of the two-phase flow model. We present the complete analytical solution to the Riemann problem (constant initial and
\end{abstract}


injected states) in detail, and describe the characteristic waves that may arise, concluding that only 9 combinations of rarefactions, shocks and rarefaction-shocks are possible. We demonstrate that assuming the saturation paths of the solution are straight lines may result in very inaccurate predictions for some realistic systems. Efficient algorithms for computing the exact solution are also given, making the analytical developments presented here readily applicable to the interpretation of lab displacement experiments, and to the implementation in streamline simulators.

KEY WORDS: flow in porous media, conservation laws, Buckley-Leverett, hyperbolic system, waves, entropy solution

\section{Introduction}

Quantitative predictions of the flow of three immiscible fluids — water, oil and gas - are required to assess many processes of great practical interest: primary oil production below bubble point and with movable water; waterfloods, man-made and natural; immiscible $\mathrm{CO}_{2}$ floods; steam floods; gravity drainage of gas caps with oil and water; water-alternating-gas (WAG) processes; and contaminant intrusions into the shallow subsurface, just to name a few.

Here we study the classical formulation of three-phase flow, which makes use of the common multiphase extension of Darcy's equation [33]. When the fractional flow approach is used, flow of three immiscible incompressible fluids is described by a pressure equation (whose solution is trivial in the one-dimensional case) and a $2 \times 2$ system of saturation equations [10]. Capillarity effects enter the formulation as a nonlinear diffusion term. It was long believed that, for negligible capillary forces, the system of equations would be strictly hyperbolic for any relative permeability functions. This is far from being the case and, in fact, most relative permeability models used today give rise to systems which are not strictly hyperbolic for the entire range of admissible saturations $[8,15,18,19,39,40]$. Loss of strict hyperbolicity typically occurs at bounded regions of the saturation triangle (the so-called elliptic regions), where the system is elliptic in character. The only relative

permeability models in the existing literature which do not produce elliptic 
regions are those where the relative permeability of a phase depends solely on the saturation of that phase $[30,44]$. This behavior is not supported by experimental results [34]. For models of this type, the elliptic region (where eigenvalues are complex conjugates) shrinks to an isolated umbilic point (a saturation state with a real double eigenvalue), thus rendering a nonstrictly hyperbolic system at that point. The literature on mixed elliptic/hyperbolic systems, and nonstrictly hyperbolic systems is vast (see, e.g., $[22,27,31]$ and the references therein), yet a complete theory is still lacking.

It is our opinion that the presence of elliptic regions contradicts the expected physical behavior of a three-phase displacement. Arguments supporting this view are given in [20]. More precisely, elliptic regions should not be justified simply because they appear as a consequence of using a questionable postulate (the multiphase extension of Darcy's equation) with common relative permeability functions. Therefore, we propose that if this questionable model is to be used, appropriate relative permeabilities should be employed, so as to avoid nonphysical behavior of the solution. It turns out [20] that it is indeed possible to impose conditions on the relative permeability functions so that the system of saturation equations is strictly hyperbolic inside the entire saturation triangle. The required conditions are in agreement with pore-scale physics and experimental data.

With the above considerations in mind, we study in this paper the solution to the Riemann problem - initial data given by two constant states separated by a single discontinuity - of three-phase flow. Because the system is strictly hyperbolic for all saturation paths of interest, we use the theory of Lax [25], as extended by Liu $[28,29]$ to systems whose characteristic fields are neither genuinely nonlinear nor linearly degenerate. A recent result for this type of systems [1] is used to limit the admissible wave structure that may arise in three-phase displacements. Identification of the admissible wave types allows us to present, for the first time, the complete catalogue of solutions to the Riemann problem of three-phase flow. Particular attention is given to the actual calculation of the analytical solution. Efficient algorithms based on a predictor-corrector strategy coupled with full Newton iteration are presented, which achieve quadratic convergence in all cases. Some of the developments are more easily understood when introduced in the context of the well-known theory of two-phase Buckley-Leverett flow [9]. For this reason, the analogy between three-phase flow and two-phase flow will be stressed throughout the paper.

An outline of the paper is as follows. In Section 2 we present the govern- 
R. Juanes and T. W. Patzek: Analytical solution to the Riemann problem ... 4

ing equations of two-phase and three-phase flow, under the Buckley-Leverett conditions. Important properties of the equations are explained in Section 3, and the character of the system is discussed. In Section 4 we present the complete set of solutions to the Riemann problem of three-phase flow. The analytical solution of the two-phase flow case is also given to motivate the developments. As an application of the analytical solution, in Section 5 we describe an example of water and gas injection into a core that is initially filled with oil and water, and we evaluate the accuracy of a common approximation of the exact solution. The main conclusions are summarized in Section 6. Algorithms for the efficient calculation of wave curves and selected solution types are compiled in the Appendix.

\section{Governing equations}

We study multiphase flow in porous media under the following assumptions:

1. One-dimensional flow

2. Immiscible fluids

3. Incompressible fluids

4. Homogeneous rigid porous medium

5. Multiphase flow extension of Darcy's law

6. Negligible gravitational effects

7. Negligible capillary pressure effects

A derivation of the governing equations was presented in [20] (see also [3,7, $10,37]$ ). In order to stress the analogy between two-phase and three-phase flow, we present here the fractional flow formulation of multiphase flow in a unified setting. By assumption 2, there is no mass transfer between phases and, therefore, one can identify components with phases. For two-phase flow we may have, for example, water $(w)$ and oil $(o)$ components. For threephase flow, we shall consider a system consisting of water $(w)$, gas $(g)$ and oil $(o)$. The one-dimensional mass conservation equation for the $\alpha$-phase is, in the absence of source terms:

$$
\partial_{t} m_{\alpha}+\partial_{x} F_{\alpha}=0, \quad 0<x<L, \quad t>0, \quad \alpha=1, \ldots, n,
$$


where $m_{\alpha}$ is the mass of the $\alpha$-phase per unit bulk volume, $F_{\alpha}$ is the mass flux of the $\alpha$-phase, $\partial_{t}(\cdot), \partial_{x}(\cdot)$, denote partial derivatives with respect to time and space, respectively, $L$ is the length of the domain, and $n$ is the number of phases. The mass of the $\alpha$-phase per unit bulk volume is:

$$
m_{\alpha}=\rho_{\alpha} S_{\alpha} \phi
$$

where $\rho_{\alpha}$ is the density of the $\alpha$-phase, $S_{\alpha}$ is the saturation of the $\alpha$-phase, and $\phi$ is the porosity. The phase densities and the porosity are assumed constant (assumptions 3 and 4, respectively). Mass fluxes are modeled using the common multiphase flow extension of Darcy's equation [33] (assumption 5). Neglecting gravitational and capillarity effects (assumptions 6 and 7), the mass flux of the $\alpha$-phase takes the form:

$$
F_{\alpha}=-\rho_{\alpha} k \lambda_{\alpha} \partial_{x} p
$$

where $k$ is the absolute permeability, $p$ is the pressure, and $\lambda_{\alpha}$ is the relative mobility of the $\alpha$-phase, defined as

$$
\lambda_{\alpha}:=\frac{k_{r \alpha}}{\mu_{\alpha}}
$$

where $k_{r \alpha}$ is the relative permeability, and $\mu_{\alpha}$ the dynamic viscosity of the $\alpha$-phase. Substituting Equations (2) and (3) into (1), the mass conservation equations read:

$$
\partial_{t} S_{\alpha}+\partial_{x}\left(-\frac{1}{\phi} k \lambda_{\alpha} \partial_{x} p\right)=0, \quad \alpha=1, \ldots, n .
$$

Equations (5) are not all independent from each other, as they have to satisfy the constraint that the fluids fill up the pore volume, $\sum_{\alpha=1}^{n} S_{\alpha} \equiv 1$. The fractional flow approach decouples the problem into a "pressure equation" and a "saturation equation" [10]. Adding the conservation equations for all phases and using the saturation constraint, we obtain the pressure equation:

$$
\partial_{x} v_{T}=0,
$$

where

$$
v_{T}:=-\frac{1}{\phi} k \lambda_{T} \partial_{x} p
$$


R. Juanes and T. W. Patzek: Analytical solution to the Riemann problem ... 6

is the total velocity, and $\lambda_{T}=\sum_{\alpha=1}^{n} \lambda_{\alpha}$ is the total mobility. The pressure equation dictates that the total velocity $v_{T}$ is at most a function of time. When gravity and capillary forces are not considered, the fractional flow of the $\alpha$-phase is simply:

$$
f_{\alpha}=\frac{\lambda_{\alpha}}{\lambda_{T}}
$$

\subsection{Two-phase flow}

With the definitions (7)-(8) above, two-phase flow is governed by a scalar saturation equation,

$$
\partial_{t} S_{w}+v_{T} \partial_{x} f_{w}=0
$$

and the algebraic constraint $S_{o}=1-S_{w}$. By definition, the water saturation cannot be negative or greater than 1 . For the purpose of this paper, saturations are understood to be renormalized [20], so that the range of saturations $[0,1]$ covers the two-phase flow region, where both phases are mobile. We use the following equivalent notation:

$$
u \equiv S_{w}, \quad f \equiv f_{w},
$$

and introduce the dimensionless space and time coordinates:

$$
\begin{aligned}
x_{D} & :=\frac{x}{L}, \\
t_{D} & :=\frac{1}{L} \int_{0}^{t} v_{T}(\bar{t}) \mathrm{d} \bar{t} .
\end{aligned}
$$

With these definitions, we write Equation (9) as:

$$
\frac{\partial u}{\partial t_{D}}+\frac{\partial f}{\partial x_{D}}=0
$$

We shall abuse notation and understand $x$ and $t$ as dimensionless space and time coordinates, and write (13) simply as:

$$
\partial_{t} u+\partial_{x} f=0 .
$$


R. Juanes and T. W. Patzek: Analytical solution to the Riemann problem ... 7

\subsection{Three-phase flow}

Three-phase flow is described by a $2 \times 2$ system of saturation equations,

$$
\partial_{t}\left(\begin{array}{c}
S_{w} \\
S_{g}
\end{array}\right)+v_{T} \partial_{x}\left(\begin{array}{c}
f_{w} \\
f_{g}
\end{array}\right)=\left(\begin{array}{l}
0 \\
0
\end{array}\right)
$$

and the algebraic constraint $S_{o}=1-S_{w}-S_{g}$. The solution is restricted to lie in the saturation triangle:

$$
\mathcal{T}:=\left\{\left(S_{w}, S_{g}\right): S_{w} \geq 0, \quad S_{g} \geq 0, \quad S_{w}+S_{g} \leq 1\right\}
$$

The saturation triangle is usually represented as a ternary diagram (see Figure 1$)$, on which the pair $\left(S_{w}, S_{g}\right)$ is represented as the triple $\left(S_{w}, S_{g}, S_{o}\right)$, where $S_{o} \equiv 1-S_{w}-S_{g}$. Defining the equivalent vector notation:

$$
\boldsymbol{u}:=\left(\begin{array}{l}
u \\
v
\end{array}\right) \equiv\left(\begin{array}{c}
S_{w} \\
S_{g}
\end{array}\right), \quad \boldsymbol{f}:=\left(\begin{array}{c}
f \\
g
\end{array}\right) \equiv\left(\begin{array}{c}
f_{w} \\
f_{g}
\end{array}\right)
$$

and understanding the space and time variables as their dimensionless counterparts (Equations (11)-(12)), the system (15) can be written in its final form:

$$
\partial_{t} \boldsymbol{u}+\partial_{x} \boldsymbol{f}=\mathbf{0}
$$

After a change of variables, the vector of unknowns $\boldsymbol{u}$ can be understood as the vector of reduced saturations, rather than actual saturations [20]. After this renormalization, the three-phase flow region —-where all three phases are mobile - covers the entire saturation triangle.

\section{Mathematical structure of the equations}

In this section we describe the mathematical structure of the equations presented in Section 2, and the behavior of solutions to these equations. We start with the simpler case of two-phase flow and then address the more complicated structure of three-phase flow. The character of Equations (14) and (18) will be determined by the functional dependence of the fractional flows:

$$
f_{\alpha}=\frac{\lambda_{\alpha}}{\lambda_{T}}=\frac{k_{r \alpha} / \mu_{\alpha}}{\sum_{\beta=1}^{n} k_{r \beta} / \mu_{\beta}} .
$$




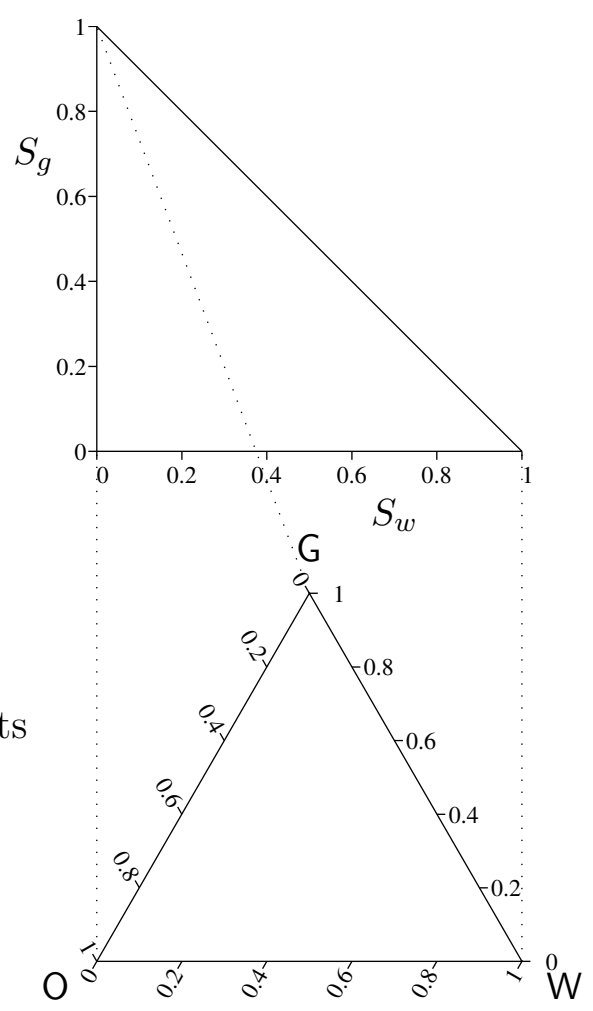

Figure 1. Saturation triangle (top) and ternary diagram (bottom).

We neglect the dependence of phase viscosities $\mu_{\alpha}$ on pressure and temperature, and take them as constants. Therefore, the character of the system is a direct consequence of the relative permeability model. Relative permeabilities $k_{r \alpha}$ are assumed to be functions of the current fluid saturations only.

\subsection{Two-phase flow}

The analysis of the scalar equation (14) governing two-phase flow is useful as a preliminary step to the much more complicated system describing threephase flow.

For two-phase flow, we assume that relative permeabilities depend on the water saturation alone:

$$
k_{r w}=k_{r w}(u), \quad k_{r o}=k_{r o}(u) .
$$


R. Juanes and T. W. Patzek: Analytical solution to the Riemann problem ... 9

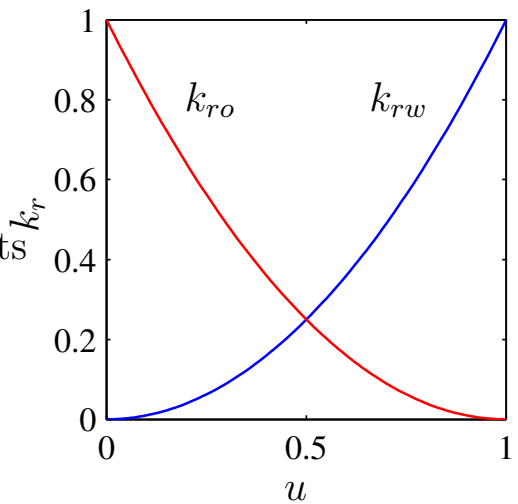

Figure 2. Typical behavior of the relative permeability functions for an oil-water system.

An example of the typically assumed behavior of relative permeabilities for an oil-water system is given by the functions (Figure 2):

$$
k_{r w}=u^{2}, \quad k_{r o}=(1-u)^{2} .
$$

From the definition of the fractional flow in Equation (8),

$$
f_{w}=\frac{k_{r w} / \mu_{w}}{\frac{k_{r w}}{\mu_{w}}+\frac{k_{r o}}{\mu_{o}}}=\frac{k_{r w}}{k_{r w}+\frac{\mu_{w}}{\mu_{o}} k_{r o}} .
$$

Using the quadratic model (20) for the relative permeabilities, and defining the viscosity ratio $\tilde{\mu}:=\mu_{w} / \mu_{o}$, we get the following expression for the flux:

$$
f(u)=\frac{u^{2}}{u^{2}+\tilde{\mu}(1-u)^{2}} .
$$

The flux function above is depicted in Figure 3 for different values of the viscosity ratio $\tilde{\mu}$. Despite its simplicity, this model displays the key features of two-phase flow in porous media: the flux function is S-shaped and, therefore, nonconvex.

Since the flux $f$ is a real function of $u$ only, the saturation equation (14) is hyperbolic. In Figure 4 we plot the flux function for the quadratic relative permeabilities (20) and a viscosity ratio $\tilde{\mu}=0.5$, and show that the saturation range $U=[0,1]$ can be split into a convex and a concave region $U=\left[0, u_{0}\right] \cup$ $\left[u_{0}, 1\right]$, respectively. Moreover, the derivative of the flux function attains its maximum value at the inflection point $u_{0}$. 
R. Juanes and T. W. Patzek: Analytical solution to the Riemann problem ... 10

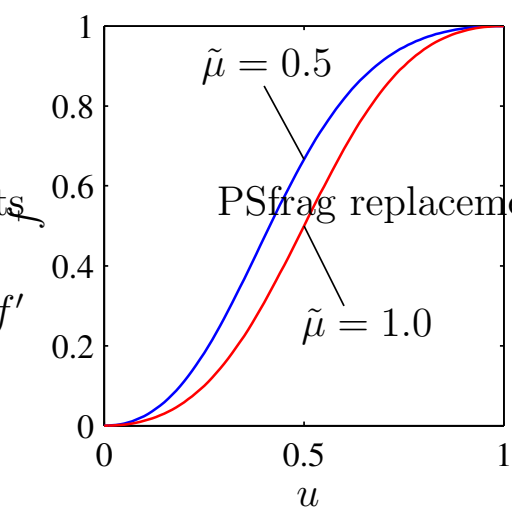

(a) Flux function

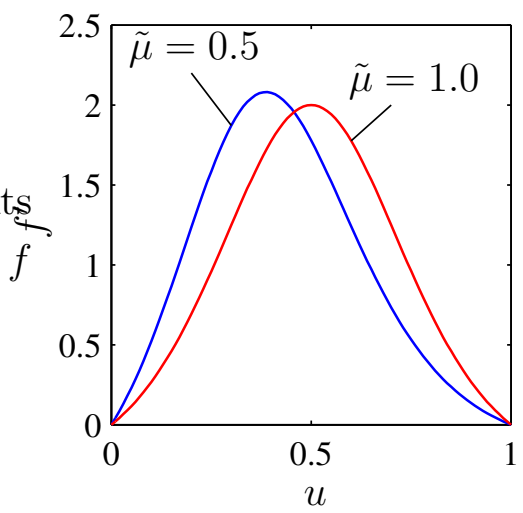

(b) Flux derivative

Figure 3. Typical behavior of the flux function in two-phase flow, for different values of the viscosity ratio $\tilde{\mu}:=\mu_{w} / \mu_{o}$.

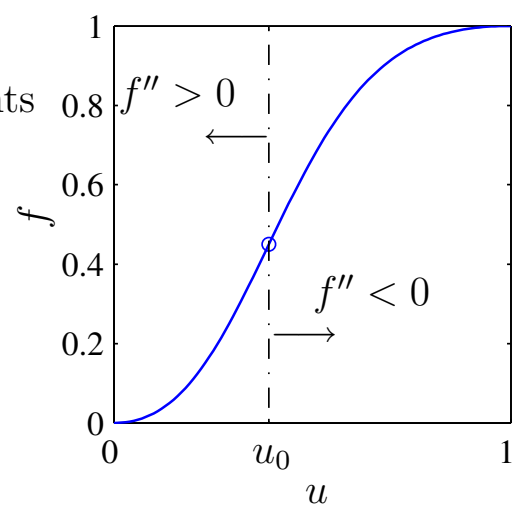

Figure 4. Convexity regions of the flux function in two-phase flow.

Remark. As we shall see, these general properties (nonconvex flux function $f$, with a single inflection point, where $f^{\prime}$ attains a maximum value) determine the type of admissible solutions of two-phase flow displacement processes.

One can use the method of characteristics to show that solutions to the two-phase saturation equation may involve discontinuities [26]. The characteristics are curves $x(t)$ on the $(x, t)$-plane, which are integral curves of the 
R. Juanes and T. W. Patzek: Analytical solution to the Riemann problem ... 11

ordinary differential equation

$$
\frac{\mathrm{d} x}{\mathrm{~d} t}=f^{\prime}(u(x, t))
$$

If Equation (14) admits a classical (continuous and differentiable) solution, the rate of change of the solution $u$ along a characteristic is

$$
\frac{D u}{D t}:=\frac{\mathrm{d}}{\mathrm{d} t} u(x(t), t)=\frac{\partial u}{\partial t}+f^{\prime}(u) \frac{\partial u}{\partial x}=0
$$

that is, $u$ is constant along the characteristics. As illustrated in Figure 5, the construction above develops a triple-valued solution even for smooth initial conditions. This non-physical behavior indicates that the classical solution has to be replaced by a weak (discontinuous) solution.

\subsection{Three-phase flow}

We now study the mathematical structure of the system of equations (18) governing three-phase flow. We argue what is the expected character of the system, and what restrictions should be imposed on the relative permeabilities as functions of fluid saturations.

\subsubsection{Character of the system of equations}

The system (18) is a $2 \times 2$ real system of first-order partial differential equations. Its character is determined by the eigenvalue problem:

$$
\boldsymbol{A r}=\nu \boldsymbol{r}
$$

where

$$
\boldsymbol{A}:=\mathrm{D}_{\boldsymbol{u}} \boldsymbol{f} \equiv \boldsymbol{f}^{\prime}(\boldsymbol{u}) \equiv\left(\begin{array}{ll}
f_{, u}(\boldsymbol{u}) & f_{, v}(\boldsymbol{u}) \\
g_{, u}(\boldsymbol{u}) & g_{, v}(\boldsymbol{u})
\end{array}\right)
$$

is the Jacobian matrix of the system, $\nu$ is an eigenvalue, and $\boldsymbol{r}$ is a right eigenvector, all evaluated at the saturation state $\boldsymbol{u}$. Subscripts after a comma denote differentiation $\left(e . g ., f_{, u} \equiv \partial_{u} f\right)$. The eigenvalues $\nu_{i}, i=1,2$ are given by:

$$
\nu_{1,2}=\frac{1}{2}\left[f_{, u}+g_{, v} \mp \sqrt{\left(f_{, u}-g_{, v}\right)^{2}+4 f_{, v} g_{, u}}\right] \text {. }
$$


R. Juanes and T. W. Patzek: Analytical solution to the Riemann problem ... 12

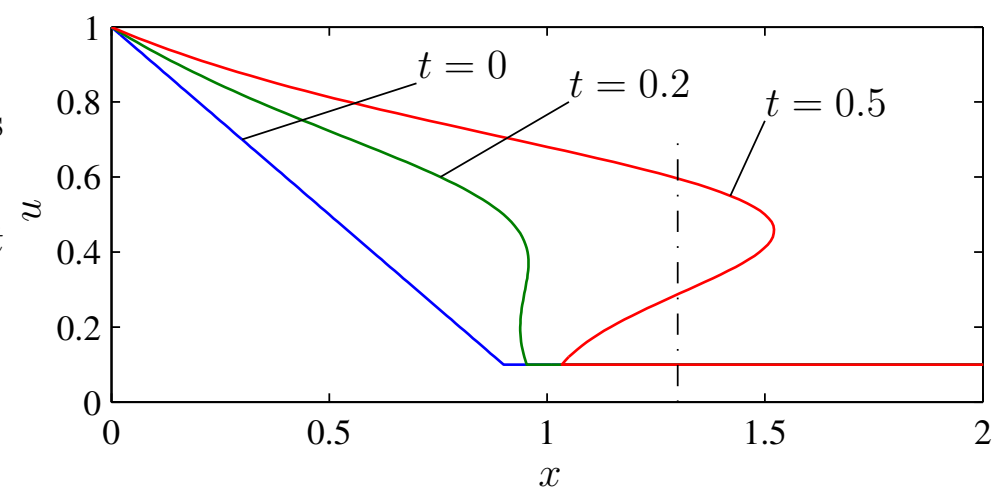

(a) Solution profile

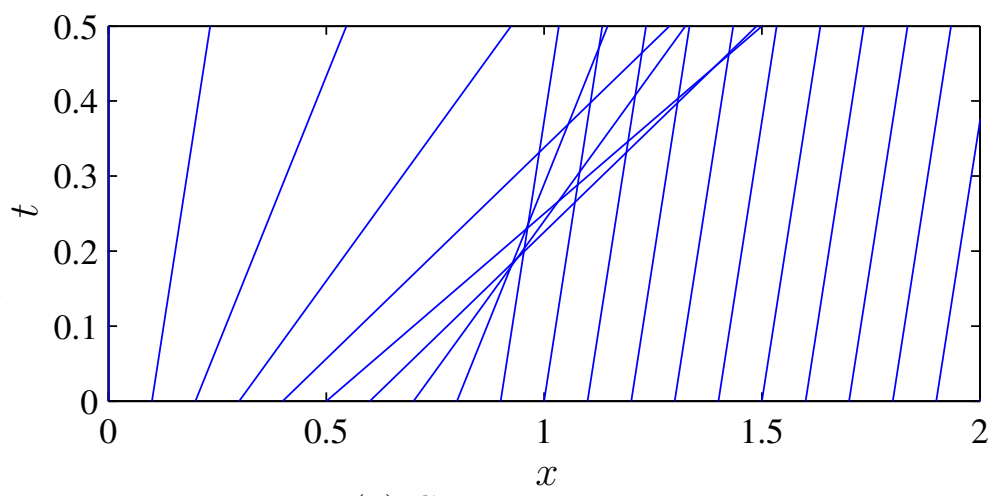

(b) Characteristics

Figure 5. The two-phase Buckley-Leverett flow problem develops a nonphysical triple-valued solution, with characteristics that intersect each other, even when the initial conditions are smooth.

The right eigenvectors $\boldsymbol{r}_{i}=\left[r_{i u}, r_{i v}\right]^{t}, i=1,2$ are calculated by the following expressions:

$$
\begin{aligned}
& \frac{r_{1 v}}{r_{1 u}}=\frac{\nu_{1}-f_{, u}}{f_{, v}}=\frac{g_{, u}}{\nu_{1}-g_{, v}}, \\
& \frac{r_{2 u}}{r_{2 v}}=\frac{f_{, v}}{\nu_{2}-f_{, u}}=\frac{\nu_{2}-g_{, v}}{g_{, u}} .
\end{aligned}
$$

The system may be classified into four categories [20]:

1. Strictly hyperbolic. The eigenvalue problem has two real, distinct, 
R. Juanes and T. W. Patzek: Analytical solution to the Riemann problem ... 13

eigenvalues. The Jacobian matrix is diagonalizable and there are two real and linearly independent eigenvectors [2].

2. Elliptic. The eigenvalues are complex conjugates.

3. Nonstrictly hyperbolic. There is a double real eigenvalue, and the Jacobian matrix is diagonalizable. The system is hyperbolic (real eigenvalues and linearly independent eigenvectors) but not strictly hyperbolic (which requires that the eigenvalues be distinct).

4. Parabolic. The system has a real, double eigenvalue, and the Jacobian matrix is defective (non-diagonalizable).

In [20] we argue that the system should be strictly hyperbolic for all saturation states in the three-phase flow region. In this case, the system has two distinct families of characteristic curves, which carry waves traveling at different characteristic speeds [47]. In particular, we do not allow for mathematical singularities such as elliptic regions (regions of the saturation space where the system is elliptic in character) and umbilic points (isolated saturation states where the system is nonstrictly hyperbolic) inside the saturation triangle. We support the view that these singularities are artifacts of an incorrect mathematical model, rather than a necessary consequence dictated by physics $[10,11,15,20,21,32,40]$.

\subsubsection{Relative permeabilities}

It is not the purpose of this paper to review the current models of relative permeabilities (see, e.g., $[4,32]$ and the references therein). It suffices to say that most of them give rise to elliptic regions $[8,15,19,40]$. In [20] we show it is possible to formulate models which are strictly hyperbolic everywhere in the three-phase flow region, even when using the usual multiphase form of Darcy's equation, and relative permeabilities which are functions of the current fluid saturations alone. Enforcing strict hyperbolicity leads to generic conditions that the relative permeability functions must satisfy. It turns out that the most serious condition that needs to be imposed is a positive derivative of the gas relative permeability near its immobile saturation. This requirement is supported by pore-scale physics and is also in good agreement with experimental data [20]. The proposed type of relative permeabilities produces a model that: (1) is everywhere strictly hyperbolic inside 
R. Juanes and T. W. Patzek: Analytical solution to the Riemann problem ... 14

the ternary diagram; and (2) presents a single umbilic point at the corner of $100 \%$ reduced gas saturation (the G vertex of the saturation triangle).

In $[20]$ we also show that a very simple relative permeability model satisfies all the required conditions. The relative mobilities are given by:

$$
\begin{aligned}
\lambda_{w} & =\left(1 / \mu_{w}\right) u^{2}, \\
\lambda_{g} & =\left(1 / \mu_{g}\right)\left(\beta_{g} v+\left(1-\beta_{g}\right) v^{2}\right), \quad \beta_{g}>0 \\
\lambda_{o} & =\left(1 / \mu_{o}\right)(1-u-v)(1-u)(1-v) .
\end{aligned}
$$

The most important feature of the model is the positive derivative of the gas relative permeability function as it approaches zero. The model considers that the relative permeabilities of the most and least wetting fluids (usually water and gas) depend only on their own saturation, whereas the relative permeability of the intermediate wetting fluid (usually oil) depends on all saturations. This is a common assumption in hydrogeology [36] and petroleum engineering $[42,43]$. Although we do not defend this assumption in general, it can be shown [20] that the relative mobilities (30)-(32) yield a system which is strictly hyperbolic everywhere in the saturation triangle, as long as the following conditions are satisfied:

$$
\begin{aligned}
\beta_{g} & >\frac{\mu_{g}}{\sqrt{\mu_{o} \mu_{w}}}, \\
\mu_{w} & <2 \mu_{o} .
\end{aligned}
$$

For illustrative purposes, we take reasonable values of the viscosities:

$$
\mu_{w}=0.875, \quad \mu_{g}=0.03, \quad \mu_{o}=2 \mathrm{cp},
$$

and a small value of the endpoint slope: $\beta_{g}=0.1$. These values of the parameters satisfy the two conditions (33)-(34) above. Relative permeabilities for all three phases are shown in Figure 6. We use this model to carry out all our sample calculations.

\subsubsection{Fractional flow functions}

From the definition (8), water and gas fractional flow functions are, respectively (see Figure 7),

$$
\begin{aligned}
& f(u, v)=\frac{\lambda_{w}(u)}{\lambda_{T}(u, v)}, \\
& g(u, v)=\frac{\lambda_{g}(v)}{\lambda_{T}(u, v)} .
\end{aligned}
$$


R. Juanes and T. W. Patzek: Analytical solution to the Riemann problem ... 15

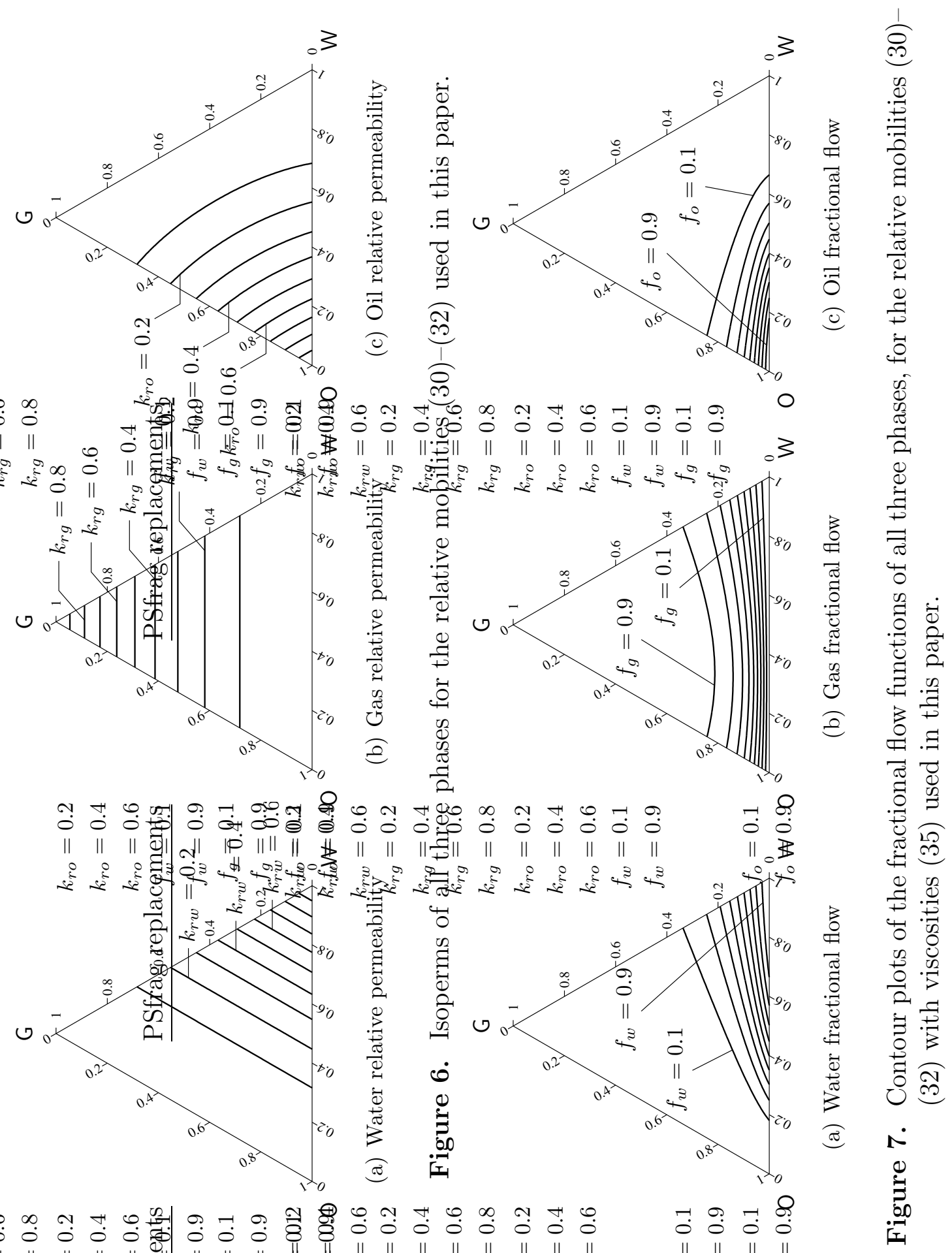


R. Juanes and T. W. Patzek: Analytical solution to the Riemann problem ... 16

\section{Solution to the Riemann problem}

\subsection{Introduction}

The Riemann problem consists in finding a (usually weak) solution to the system of conservation laws:

$$
\partial_{t} \boldsymbol{u}+\partial_{x} \boldsymbol{f}=\mathbf{0}, \quad-\infty<x<\infty, t>0,
$$

with initial condition

$$
\boldsymbol{u}(x, 0)= \begin{cases}\boldsymbol{u}_{l} & \text { if } x<0, \\ \boldsymbol{u}_{r} & \text { if } x>0 .\end{cases}
$$

For three-phase flow, the system of governing equations is the $2 \times 2$ system (18), whereas for two-phase flow it reduces to the scalar equation (14). Unrealistic as it may seem (unbounded domain, and piecewise constant initial data with a single discontinuity), the solution to the Riemann problem is extremely valuable for practical applications. Many laboratory experiments reproduce in fact the conditions of the Riemann problem: the medium has initially homogeneous saturations, and the proportion of injected fluids is held constant during the experiment. The solution to the Riemann problem gives also information about the structure of the system of equations, and can be used as the building block for obtaining solutions to problems with more complex initial conditions (as in the Godunov method [16,26]).

The system (38) is invariant under uniform stretching of coordinates $(x, t) \mapsto(c x, c t)$ and, therefore, admits self-similar solutions. The property of self-similarity has been termed "stretching principle" [45] or "coherence condition" $[17,38]$ in the petroleum engineering literature. It means that the solution at different times "can be obtained from one another by a similarity transformation." [5] We seek a solution of the form

$$
\boldsymbol{u}(x, t)=\boldsymbol{U}(\zeta)
$$

where, in our case, the similarity variable $\zeta$ is simply

$$
\zeta:=\frac{x}{t}
$$

It can be shown (see, e.g., [12]) that self-similar solutions (40) of the Riemann problem (38)-(39) are composites of constant states, shocks joining 
constant states, and rarefaction waves connecting constant states or contact discontinuities. More precisely, since for a strictly hyperbolic system waves of different families are strictly separated, any self-similar solution to the Riemann problem for a $n \times n$ system comprises $n+1$ constant states:

$$
\boldsymbol{u}_{l}=\boldsymbol{u}_{0} \stackrel{\mathcal{W}_{1}}{\longrightarrow} \boldsymbol{u}_{1} \stackrel{\mathcal{W}_{2}}{\longrightarrow} \cdots \stackrel{\mathcal{W}_{n-1}}{\longrightarrow} \boldsymbol{u}_{n-1} \stackrel{\mathcal{W}_{n}}{\longrightarrow} \boldsymbol{u}_{n}=\boldsymbol{u}_{r}
$$

States $\boldsymbol{u}_{i-1}$ and $\boldsymbol{u}_{i}$ are joined by a wave of the $i$-family $\left(\mathcal{W}_{i}\right)$ which, in general, may consist of $i$-rarefactions, $i$-shocks and/or $i$-contact discontinuities.

Next, the admissible wave structure for two-phase and three-phase flow is described, and the complete set of solutions to the Riemann problem is given.

\subsection{Riemann problem for two-phase flow}

The solution to the two-phase displacement problem was presented originally by Buckley and Leverett [9]. Many of the features in the displacement theory

of three-phase flow are natural extensions of concepts already present in the two-phase flow case. Some concepts are more easily understood in the latter case, which involves a scalar equation rather than a system.

\subsubsection{Wave structure}

In the two-phase flow case, the Riemann problem involves the scalar equation (14). Therefore, the left and right states $\left(u_{l}\right.$ and $u_{r}$, respectively) are joined by a single wave:

$$
u_{l} \stackrel{\mathcal{W}}{\longrightarrow} u_{r}
$$

When the flux function $f$ of a scalar conservation law is convex, the wave appearing in the solution is either a shock or a rarefaction. When $f$ is not convex, as in the present case (see Figure 4), the characteristic wave may involve both [26]. We now study in more detail the structure of the wave connecting the two constant states $u_{l}$ (left) and $u_{r}$ (right). There are three possible wave types, namely: a single rarefaction, a single shock, and a composite rarefaction-shock.

Single rarefaction $(\mathcal{R})$ A rarefaction (a term coined in the context of gas dynamics) is a smooth solution $U(\zeta)$, with $U\left(\zeta_{l}\right)=u_{l}$ and $U\left(\zeta_{r}\right)=$ 
R. Juanes and T. W. Patzek: Analytical solution to the Riemann problem ... 18
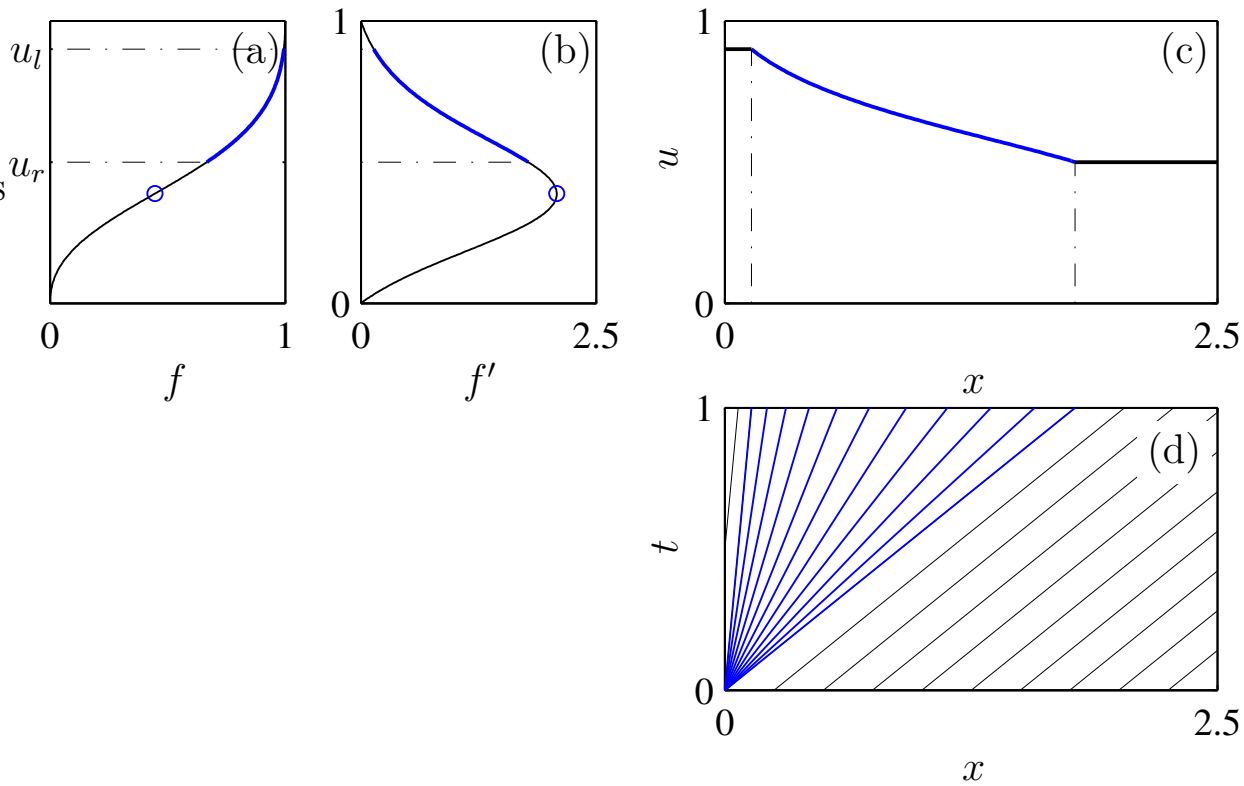

Figure 8. Example of a single rarefaction solution to the Riemann problem of two-phase flow. The characteristic speed $f^{\prime}$ increases monotonically from the left state to the right state, and the solution is smooth and single-valued.

$u_{r}$. If the solution is smooth (continuous and differentiable), substituting Equations (40)-(41) into Equation (14), we obtain:

$$
f^{\prime}(U) U^{\prime}=\zeta U^{\prime}
$$

If the solution is not a constant function, that is, if $U^{\prime} \neq 0$, it must satisfy:

$$
\zeta=f^{\prime}(U)
$$

Clearly, the solution is admissible only if the characteristic speed $f^{\prime}$ increases monotonically from the left state to the right state. Otherwise, characteristics intersect on the $x$-t plane, and the solution is not single-valued. For the characteristic speed to increase monotonically, both left and right states must lie on the same convexity region, that is,

$$
u_{l}>u_{r} \geq u_{0}, \quad \text { or } \quad u_{l}<u_{r} \leq u_{0}
$$


R. Juanes and T. W. Patzek: Analytical solution to the Riemann problem ... 19

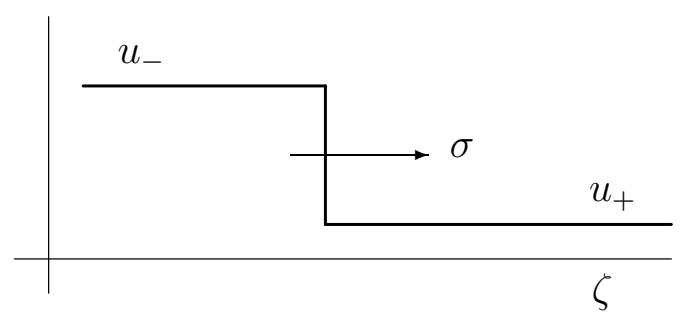

Figure 9. Schematic of a shock.

An example of a rarefaction wave solution is shown in Figure 8 for the case $u_{l}>u_{r} \geq u_{0}$, using the fractional flow function (22) with $\tilde{\mu}=0.5$. The figure includes a plot of: (a) the flux function indicating the left and right states; (b) the flux derivative; (c) the solution profile at $t=1$; and (d) the characteristics on the $x$ - $t$ plane. The solution is constant along characteristics, and characteristics spread from the origin in a rarefaction fan.

Single shock $(\mathcal{S})$ A shock is a traveling discontinuity. Discontinuities are allowed in the context of weak solutions, and they develop whenever the characteristic speed $f^{\prime}$ at the left state is larger than that at the right state. Otherwise, characteristics would intersect and the solution would be multiple-valued. If a shock connects two states, $u_{-}=U\left(\zeta_{-}\right)$and $u_{+}=U\left(\zeta_{+}\right)$, as shown schematically in Figure 9, the speed of propagation $\sigma$ is determined by the Rankine-Hugoniot condition:

$$
f\left(u_{+}\right)-f\left(u_{-}\right)=\sigma \cdot\left(u_{+}-u_{-}\right)
$$

This condition is, in essence, a mass conservation statement when the solution is discontinuous. In the scalar case, it is equivalent to the equal-area rule $[9$, 26]. For the solution to be admissible, the shock must satisfy the Lax entropy condition for genuine shocks $[25,26,41]$ :

$$
f^{\prime}\left(u_{-}\right)>\sigma>f^{\prime}\left(u_{+}\right)
$$

In Figure 10 we show the case of a single shock between two constant states, including: (a) the flux function indicating the left and right states; (b) the flux derivative and the shock speed; (c) the solution profile at $t=1$; and $(\mathrm{d})$ the characteristics on the $x-t$ plane. The solution satisfies the Lax entropy condition, which implies that characteristics go into the shock. 
R. Juanes and T. W. Patzek: Analytical solution to the Riemann problem ... 20
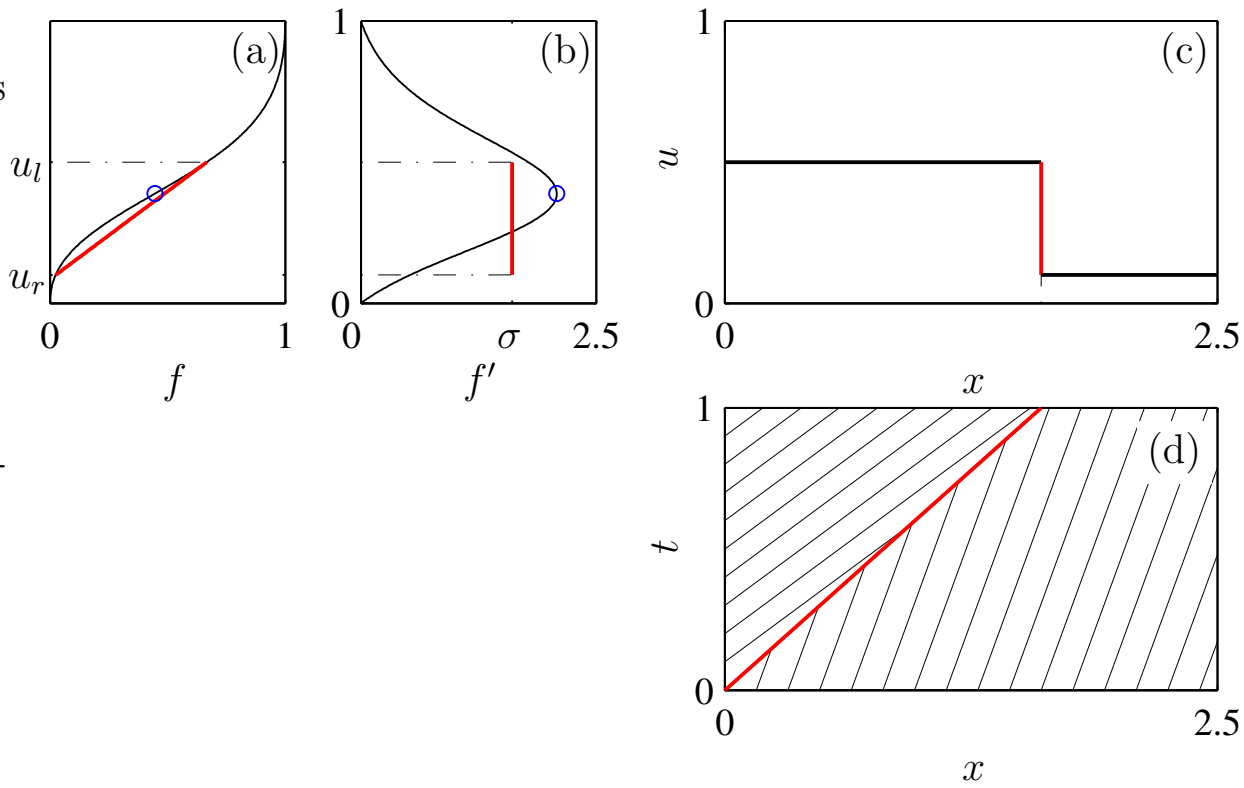

Figure 10. Example of a single shock solution to the Riemann problem of two-phase flow. The speed of propagation of the discontinuity is determined by the Rankine-Hugoniot condition, and it is readily checked that characteristics go into the shock.

Composite rarefaction-shock $(\mathcal{R S})$ It might not be possible to connect left and right states with a simple wave. In some cases, a composite wave consisting of a rarefaction and a shock is required. The left and right states must lie on different convexity regions so that the characteristic speed is not monotonic, that is,

$$
u_{l}>u_{0}>u_{r}, \quad \text { or } \quad u_{l}<u_{0}<u_{r}
$$

The solution involves at most one rarefaction and one shock, because the fractional flow function has one inflection point only. Moreover, since the inflection point corresponds to a maximum value of the derivative, the rarefaction fan of a composite wave is always slower than the shock [1]. The rarefaction and the shock are connected at some intermediate point $u_{*}$, called the post-shock value. This is the value of $u$ at which the left characteristic speed (rarefaction fan) coincides with the speed of the right discontinuity 
R. Juanes and T. W. Patzek: Analytical solution to the Riemann problem ... 21
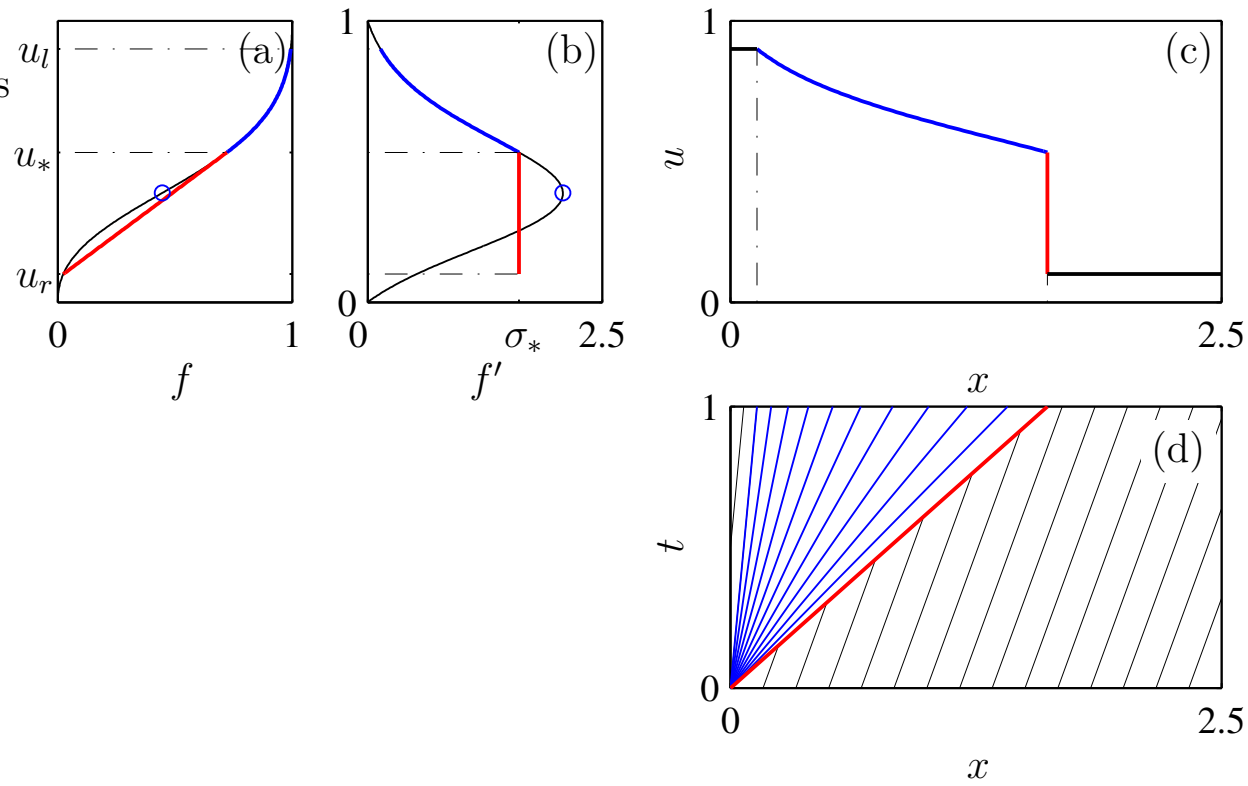

Figure 11. Example of a composite rarefaction-shock solution to the Riemann problem of two-phase flow. Left and right states lie necessarily on opposite sides of the inflection point and the rarefaction is always behind the shock. The speed of propagation of the discontinuity is determined by the concave hull construction.

(shock):

$$
f^{\prime}\left(u_{*}\right)=\sigma_{*}:=\frac{f\left(u_{*}\right)-f\left(u_{r}\right)}{u_{*}-u_{r}} .
$$

Equation (50) is a nonlinear equation, which can be solved, for example, by Newton iteration.

The wave joining the left and right constant states is, thus, a rarefactionshock $(\mathcal{W} \equiv \mathcal{R S})$, which we express schematically as follows:

$$
u_{l} \stackrel{\mathcal{R}}{\longrightarrow} u_{*} \stackrel{\mathcal{S}}{\longrightarrow} u_{r}
$$

The appropriate criterion for ascertaining admissible shocks when the flux function is not convex is the Oleinik entropy condition [12,35,41], which 
R. Juanes and T. W. Patzek: Analytical solution to the Riemann problem ... 22

states that any discontinuity joining the states $u_{-}$and $u_{+}$must satisfy:

$$
\frac{f(u)-f\left(u_{-}\right)}{u-u_{-}} \geq \frac{f\left(u_{+}\right)-f\left(u_{-}\right)}{u_{+}-u_{-}} \geq \frac{f\left(u_{+}\right)-f(u)}{u_{+}-u},
$$

for all $u$ between $u_{-}$and $u_{+}$. This entropy criterion is equivalent to the concave hull construction [26]. In the particular case of two-phase flow, where the flux function has only one change in convexity, associated with a maximum value of the derivative, condition (52) reduces to checking that the characteristic speed $f^{\prime}$ must increase from left to right along the rarefaction fan, and that the shock satisfies:

$$
f^{\prime}\left(u_{*}\right) \geq \sigma_{*}>f^{\prime}\left(u_{r}\right)
$$

A solution which involves a composite rarefaction-shock wave is shown in Figure 11, computed for the fractional flow function (22) with $\tilde{\mu}=0.5$, as before.

\subsubsection{Complete set of solutions}

The solution to the Riemann problem of two-phase flow consists in a single wave that joins the left and right constant states. As shown above, this wave may only be a rarefaction, a shock, or a rarefaction-shock. Therefore, only three types of solutions are possible. In Figure 12 we present an algorithm which summarizes the process of obtaining the wave structure for two-phase flow.

\subsection{Riemann problem for three-phase flow}

The system of conservation laws (18) describing three-phase flow is a $2 \times 2$ system, which is strictly hyperbolic for all saturation paths of interest [20]. This implies that there are two separated waves connecting three constant states: $\boldsymbol{u}_{l}$ (left), $\boldsymbol{u}_{m}$ (middle), and $\boldsymbol{u}_{r}$ (right). Therefore, the solution to the Riemann problem for three-phase flow reduces to finding the intermediate constant state $\boldsymbol{u}_{m}$ as the intersection of an admissible 1-wave $\mathcal{W}_{1}$ (slow wave) and an admissible 2 -wave $\mathcal{W}_{2}$ (fast wave) on the saturation triangle (Figure 13):

$$
\boldsymbol{u}_{l} \stackrel{\mathcal{W}_{1}}{\longrightarrow} \boldsymbol{u}_{m} \stackrel{\mathcal{W}_{2}}{\longrightarrow} \boldsymbol{u}_{r}
$$


R. Juanes and T. W. Patzek: Analytical solution to the Riemann problem ... 23

- Given left and right states: $u_{l}, u_{r}$

- Trial shock speed: $\sigma^{\text {trial }}=\frac{f\left(u_{l}\right)-f\left(u_{r}\right)}{u_{l}-u_{r}}$

IF $f^{\prime}\left(u_{l}\right)>\sigma^{\text {trial }}>f^{\prime}\left(u_{r}\right)$ THEN

- $\mathcal{S}$ : Single shock with speed $\sigma=\sigma^{\text {trial }}$

ELSE

IF $f^{\prime}\left(u_{l}\right)<f^{\prime}\left(u_{r}\right) \& f^{\prime \prime}\left(u_{l}\right) f^{\prime \prime}\left(u_{r}\right)>0$ THEN

- $\mathcal{R}$ : Single rarefaction

ELSE

- $\mathcal{R S}$ : Composite rarefaction-shock

- Post-shock value $u_{*}$ such that $f^{\prime}\left(u_{*}\right)=\sigma_{*}$

- Shock speed: $\sigma_{*}=\frac{f\left(u_{*}\right)-f\left(u_{r}\right)}{u_{*}-u_{r}}$

END

END

Figure 12. Algorithm for obtaining the wave structure for two-phase flow.

The theory of strictly hyperbolic systems was compiled by Lax [25]. The solution to the Riemann problem was restricted to systems whose characteristic fields are either genuinely nonlinear or linearly degenerate. The notion

of genuine nonlinearity, which is made precise below, is central to solving the Riemann problem of three-phase flow. Basically, genuine nonlinearity of a characteristic field is the natural extension of convexity of the flux function for scalar equations. As we shall see, this property does not hold for the three-phase flow system, much in the same way as the flux function in twophase flow is not convex. The theory of Lax was extended by Liu $[28,29]$ to find Riemann solutions for systems with nongenuinely nonlinear fields. This theory is used here to describe the admissible wave structure, and the complete set of solutions to the Riemann problem. 
R. Juanes and T. W. Patzek: Analytical solution to the Riemann problem ... 24

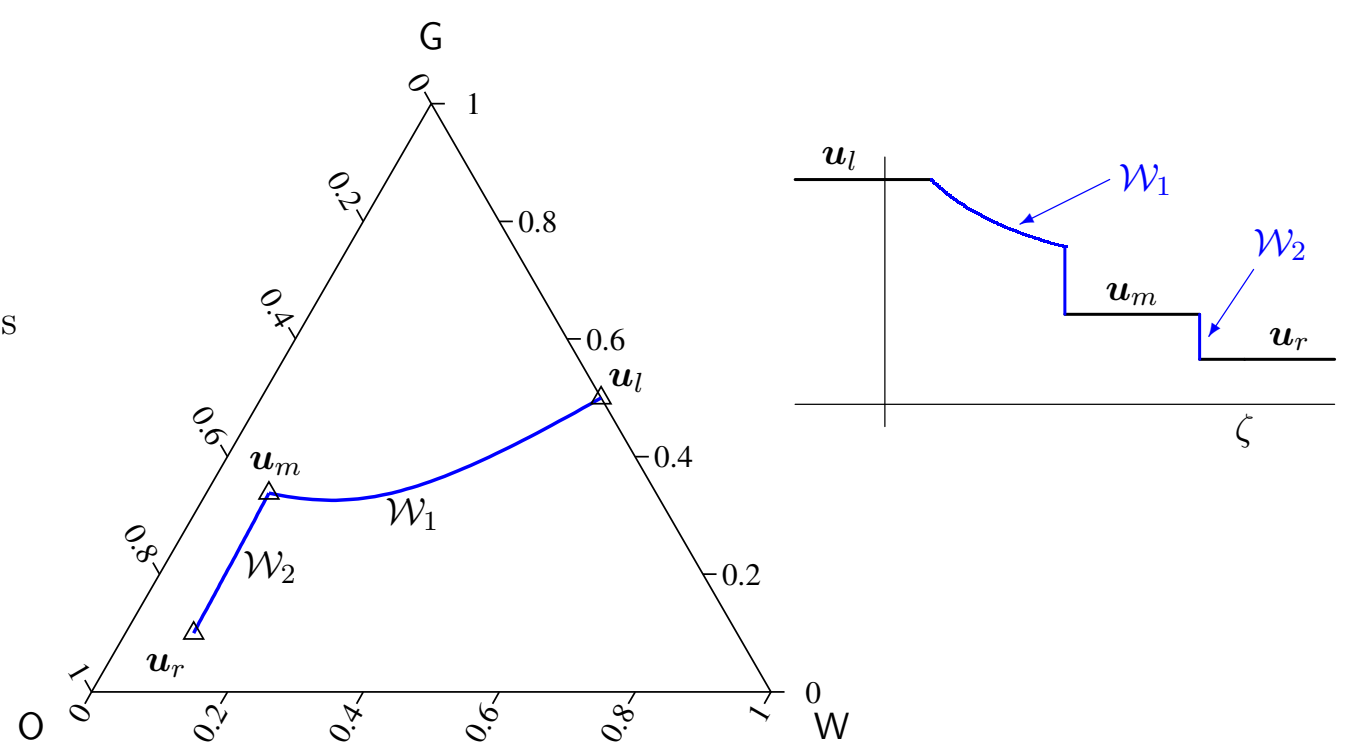

Figure 13. Schematic representation of the generic solution to the Riemann problem of three-phase flow. The solution comprises two distinct waves (slow and fast waves) connecting three constant states, and each wave might involve traveling discontinuities. On the left plot we show a possible configuration of the wave curves in the saturation space. On the right plot we display the corresponding saturation profile for one of the components (gas saturation, say) against the similarity variable $\zeta=x / t$.

\subsubsection{Wave structure}

We now describe the structure of the waves in the Riemann solution. From the theory of strictly hyperbolic conservation laws [12], a wave of the $i$-family consists of $i$-rarefactions, $i$-shocks and/or $i$-contact discontinuities. This is discussed next.

Integral curves and rarefactions Rarefactions are smooth waves joining constant states or contact discontinuities. If the solution is smooth, using Equations (40)-(41) in Equation (38), a self-similar solution of the Riemann problem satisfies the system of ordinary differential equations

$$
\boldsymbol{A}(\boldsymbol{U}) \boldsymbol{U}^{\prime}=\zeta \boldsymbol{U}^{\prime}
$$


R. Juanes and T. W. Patzek: Analytical solution to the Riemann problem . . 25

where $\boldsymbol{A}(\boldsymbol{U})$ is the Jacobian matrix of the system (Equation (26)). This is an eigenvalue problem, where the similarity variable $\zeta=x / t$ is an eigenvalue, and $\boldsymbol{U}^{\prime}$ is a right eigenvector. Because the system is strictly hyperbolic (Section 3.2.1), there exist two distinct eigenvalues $\nu_{i}$ and two linearly independent eigenvectors $\boldsymbol{r}_{i}$, corresponding to the two different characteristic families $i=1,2$. This leads to the following definition: An $i$-rarefaction is a smooth function $\boldsymbol{U}_{i}(\zeta)$ satisfying Equation (55), where the parameter $\zeta$ is not arbitrary, but the $i$-eigenvalue of the Jacobian matrix of the system:

$$
\zeta=\nu_{i}\left(\boldsymbol{U}_{i}(\zeta)\right)
$$

It follows that an $i$-rarefaction curve (in phase space) must lie on an integral curve of the $i$-family, that is, a curve whose tangent at any point $\boldsymbol{U}$ is in the direction of the $i$-eigenvector $\boldsymbol{r}_{i}(\boldsymbol{U})$ at that point. The two families of integral curves, usually termed as slow and fast paths, are depicted in Figure 14 for the relative mobilities (30)-(32).

A rarefaction curve $\boldsymbol{U}_{i}(\zeta)$ will provide an admissible single-valued solution only if the similarity variable parameter $\zeta=\nu_{i}$ increases monotonically along the curve from the left state to the right state.

Rarefaction curves can be calculated by simple numerical integration with a Runge-Kutta algorithm, as explained in Appendix A.1.

Hugoniot loci and shocks Any propagating discontinuity connecting two states $\boldsymbol{u}_{-}=\boldsymbol{U}\left(\zeta_{-}\right)$and $\boldsymbol{u}_{+}=\boldsymbol{U}\left(\zeta_{+}\right)$, must satisfy an integral conservation equation for each variable, known as the Rankine-Hugoniot jump condition [26]:

$$
\boldsymbol{f}\left(\boldsymbol{u}_{+}\right)-\boldsymbol{f}\left(\boldsymbol{u}_{-}\right)=\sigma\left(\boldsymbol{u}_{+} ; \boldsymbol{u}_{-}\right) \cdot\left(\boldsymbol{u}_{+}-\boldsymbol{u}_{-}\right),
$$

where $\sigma\left(\boldsymbol{u}_{+} ; \boldsymbol{u}_{-}\right)$is the speed of propagation of the discontinuity. For a fixed state $\boldsymbol{u}_{-}$, one can determine the set of states $\boldsymbol{u}_{+}$which can be connected to $\boldsymbol{u}_{-}$such that Equation (57) is satisfied. There are two families of solutions, one for each characteristic family, which form two curves passing through the reference state $\boldsymbol{u}_{-}: \mathcal{H}_{1}\left(\boldsymbol{u}_{-}\right)$and $\mathcal{H}_{2}\left(\boldsymbol{u}_{-}\right)$(Figure 15 ). The set of points on each of these curves is called the Hugoniot locus. It is easy to show [26] that the Hugoniot curves are tangent to the corresponding eigenvectors at the reference point $\boldsymbol{u}_{-}$. Moreover, since the system is strictly hyperbolic, Hugoniot loci do not have detached branches and are transversal to each other [12]. 
R. Juanes and T. W. Patzek: Analytical solution to the Riemann problem ... 26

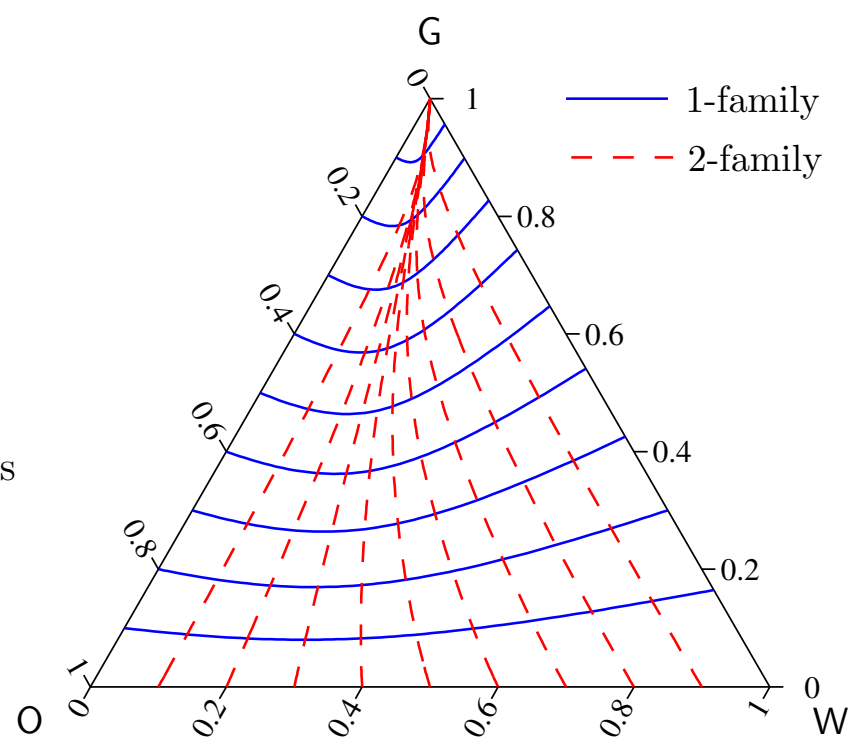

Figure 14. Integral curves for the relative mobilities (30)-(32). Integral curves of the 1- and 2-family are usually termed slow and fast paths, respectively.

Not every discontinuity satisfying the Rankine-Hugoniot condition is a valid shock. For a genuine shock of the $i$-family (an $i$-shock) to be physically admissible, it must satisfy the Lax entropy condition [12,25, 26, 41]:

$$
\nu_{i}\left(\boldsymbol{u}_{-}\right)>\sigma_{i}\left(\boldsymbol{u}_{+} ; \boldsymbol{u}_{-}\right)>\nu_{i}\left(\boldsymbol{u}_{+}\right),
$$

where $\boldsymbol{u}_{-}$and $\boldsymbol{u}_{+}$are the values at the left and at the right of the discontinuity, respectively. Condition (58) implies that characteristics of the $i$-family go into the shock. A shock curve of the $i$-family passing through point $\boldsymbol{u}_{-}$, denoted as $\mathcal{S}_{i}\left(\boldsymbol{u}_{-}\right)$, corresponds to a subset of the Hugoniot locus $\mathcal{H}_{i}\left(\boldsymbol{u}_{-}\right)$, for which the entropy condition (58) is satisfied.

An algorithmic procedure for the calculation of the Hugoniot loci, based on a Newton iterative scheme, is detailed in Appendix A.2.

Inflection loci and rarefaction-shocks The notion of genuine nonlinearity is crucial to the wave structure arising in multiphase flow. The $i$-field is said to be genuinely nonlinear if the $i$-eigenvalue $\nu_{i}$ varies monotonically 
R. Juanes and T. W. Patzek: Analytical solution to the Riemann problem . . 27

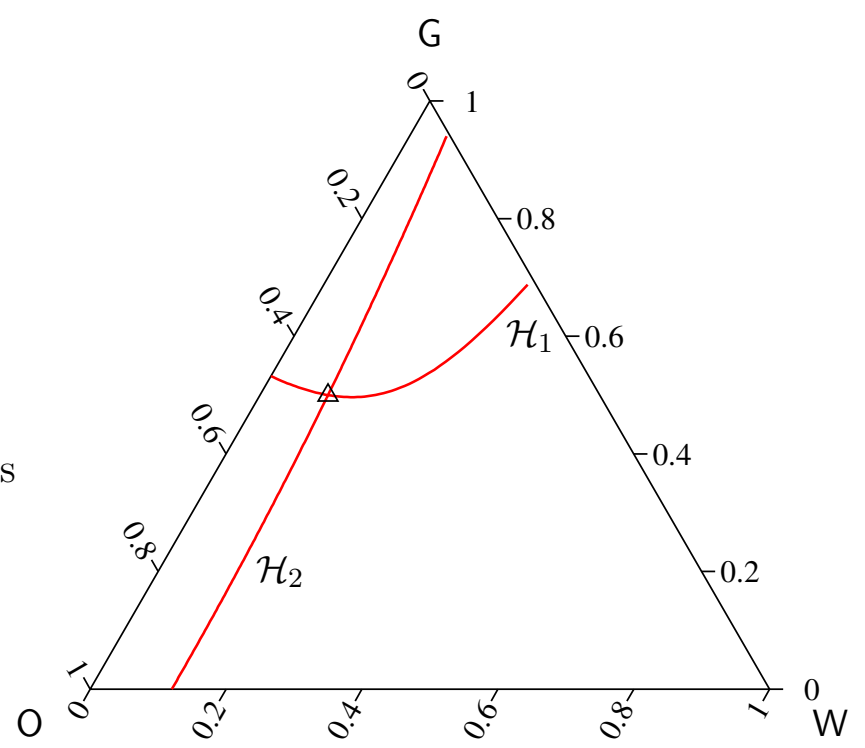

(a) Reference state $\boldsymbol{u}_{-}=(0.1,0.5)$

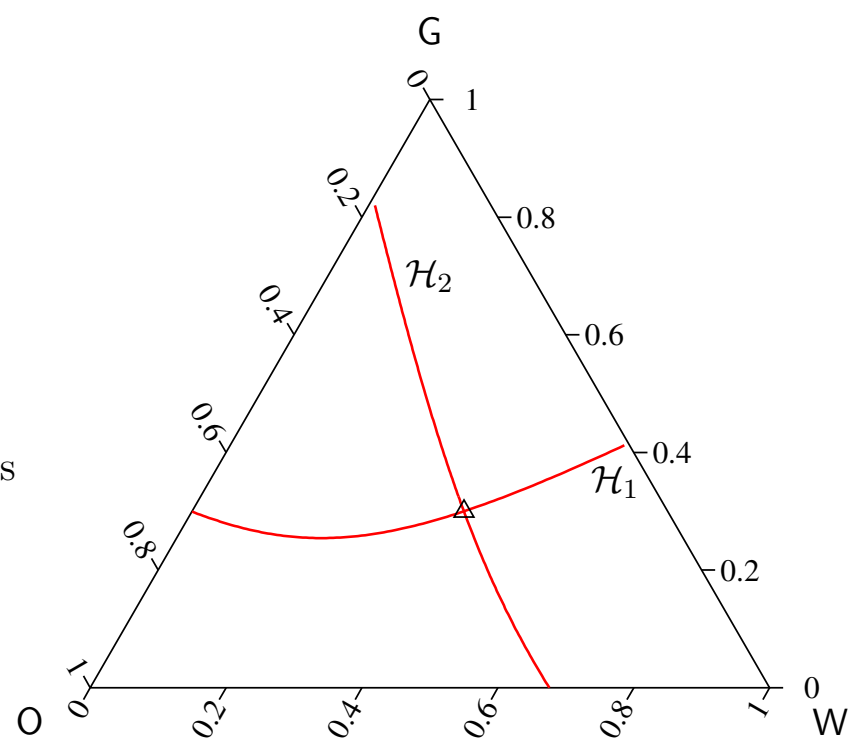

(b) Reference state $\boldsymbol{u}_{-}=(0.4,0.3)$

Figure 15. Plot of the Hugoniot loci of both characteristic families, $\mathcal{H}_{1}\left(\boldsymbol{u}_{-}\right)$ and $\mathcal{H}_{2}\left(\boldsymbol{u}_{-}\right)$, for the relative mobilities (30)-(32) and two different reference states $\boldsymbol{u}_{-}(\triangle)$. 
R. Juanes and T. W. Patzek: Analytical solution to the Riemann problem ... 28

along integral curves of the $i$-family. This is expressed mathematically as:

$$
\nabla \nu_{i}(\boldsymbol{U}) \cdot \boldsymbol{r}_{i}(\boldsymbol{U}) \neq 0 \text { for all } \boldsymbol{U}
$$

where $\nabla \nu_{i}(\boldsymbol{U}):=\left[\partial \nu_{i} / \partial u, \partial \nu_{i} / \partial v\right]^{t}$ is the gradient of $\nu_{i}(\boldsymbol{U})$. This condition is equivalent to that of convexity, $f^{\prime \prime}(u) \neq 0 \forall u$, for scalar conservation laws. The $i$-field is said to be linearly degenerate if $\nu_{i}$ is constant along integral curves of the $i$-family, that is,

$$
\nabla \nu_{i}(\boldsymbol{U}) \cdot \boldsymbol{r}_{i}(\boldsymbol{U}) \equiv 0 \quad \text { for all } \boldsymbol{U}
$$

Of course, the value of $\nu_{i}(\boldsymbol{U})$ may vary from one integral curve to the next. As it turns out, the characteristic fields of the system describing three-phase flow are neither genuinely nonlinear nor linearly degenerate: eigenvalues attain local maxima along integral curves. We can therefore define the inflection locus $\mathcal{V}_{i}$ for the $i$-characteristic field as the set of points $\boldsymbol{U}$ such that

$$
\nabla \nu_{i}(\boldsymbol{U}) \cdot \boldsymbol{r}_{i}(\boldsymbol{U})=0
$$

that is, the locations at which $\nu_{i}$ attain either a maximum or a minimum value when moving along integral curves of the $i$-family.

In Figure 16 we show contour plots of eigenvalues and the inflection loci for both characteristic families. We note that in all realistic models of multiphase flow, the inflection locus corresponds to maxima of eigenvalues. This is consistent with the well-known behavior of the flux function for the two-phase flow case, where the fractional flow function is S-shaped, and the inflection point corresponds to the maximum value of the derivative (Figure 4).

For a strictly hyperbolic system whose characteristic fields are genuinely nonlinear, any wave connecting two constant states $\boldsymbol{u}_{l}$ and $\boldsymbol{u}_{r}$ can only be a rarefaction or a genuine shock, and any discontinuity must satisfy the Lax entropy condition (58). If the characteristic fields are nongenuinely nonlinear, each wave might consist in a combination of rarefactions and discontinuities $[28,29]$. In our case, since the inflection locus for each field is a single connected curve satisfying certain orthogonality conditions with respect to integral curves, the composite wave has at most one rarefaction and one discontinuity. Moreover, because the inflection loci correspond to local maxima of eigenvalues along integral curves, the rarefaction is always slower than the shock [1].

More precisely, a rarefaction-shock of the $i$-family connecting the left and right states $\boldsymbol{u}_{l}$ and $\boldsymbol{u}_{r}$, respectively, is a curve on the phase plane consisting 
R. Juanes and T. W. Patzek: Analytical solution to the Riemann problem ... 29

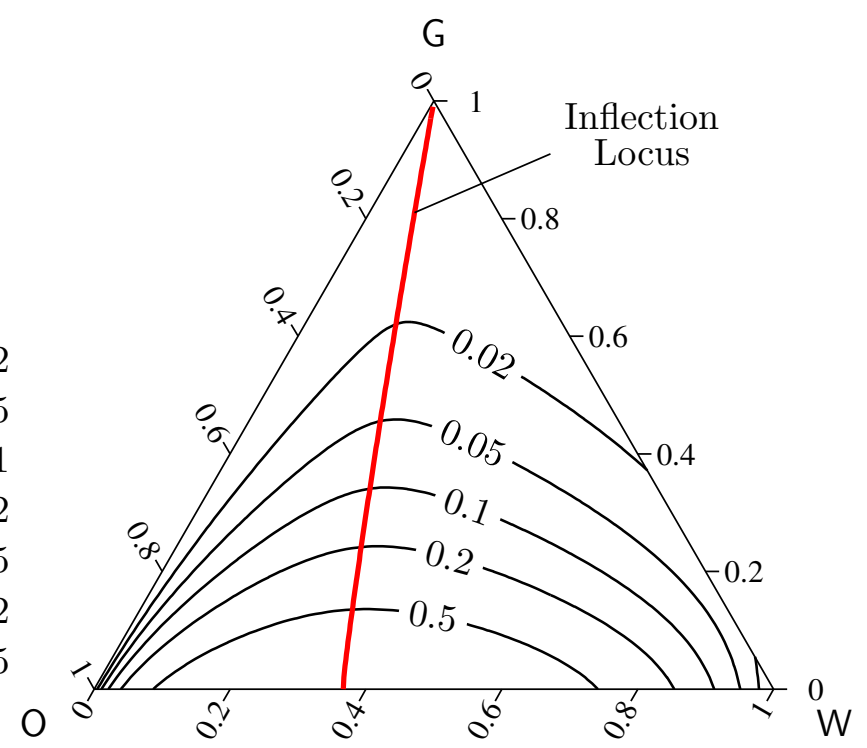

(a) 1-family

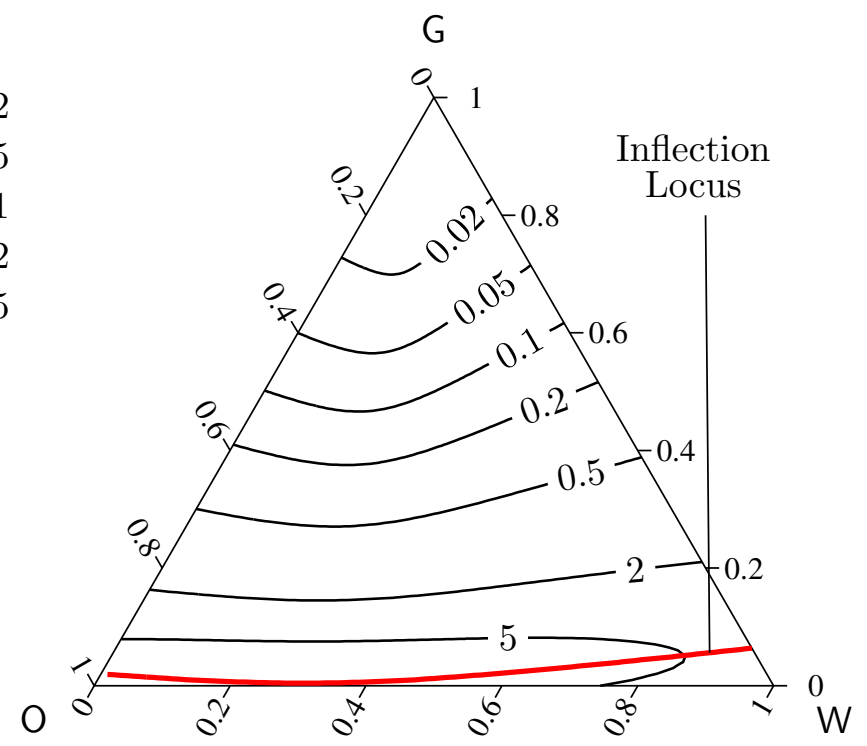

(b) 2-family

Figure 16. Contour plots of eigenvalues $\nu_{i}$ and the inflection loci $\mathcal{V}_{i}$ of both characteristic families, for the relative mobilities (30)-(32). Inflection loci correspond to local maxima of eigenvalues when moving along integral curves. 
R. Juanes and T. W. Patzek: Analytical solution to the Riemann problem . . 30

of an $i$-rarefaction curve emanating from $\boldsymbol{u}_{l}$, connected to an $i$-shock curve at some intermediate point $\boldsymbol{u}_{*}$, which ends at the right state. We denote this rarefaction-shock curve as $\mathcal{R}_{i} \mathcal{S}_{i}\left(\boldsymbol{u}_{l}, \boldsymbol{u}_{r}\right)$ and, unlike rarefaction curves or shock curves alone, is defined through both endpoints. The intermediate state $\boldsymbol{u}_{*}$ is the post-shock state, at which the following property holds:

$$
\nu_{i}\left(\boldsymbol{u}_{*}\right)=\sigma_{i}\left(\boldsymbol{u}_{r} ; \boldsymbol{u}_{*}\right) .
$$

A necessary condition for a $\mathcal{R}_{i} \mathcal{S}_{i}\left(\boldsymbol{u}_{l}, \boldsymbol{u}_{r}\right)$ wave is that the left and right states lie on opposite sides with respect to the inflection locus $\mathcal{V}_{i}$. This rules out the possibility of two such states being connected by a rarefaction wave, since the characteristic speed would not be monotonically increasing and, as a result, the solution would not be single-valued. Figure 17 shows two rarefaction-shock curves for the first characteristic family, corresponding to the same left state but two different right states. Note that the post-shock value $\boldsymbol{u}_{*}$, at which the $\mathcal{R}_{1}$ and $\mathcal{S}_{1}$ curves are connected, is different for each case. This connection is always very smooth. In fact, it can be shown [25] that both curves are connected with second order tangency (same slope and curvature).

If the $i$-characteristic field is nongenuinely nonlinear, any discontinuity in the $i$-wave, connecting two states $\boldsymbol{u}_{-}$(left) and $\boldsymbol{u}_{+}$(right), must satisfy the Liu entropy condition $[28,29]$, which states that

$$
\sigma_{i}\left(\boldsymbol{u}_{+} ; \boldsymbol{u}_{-}\right) \leq \sigma_{i}\left(\boldsymbol{u} ; \boldsymbol{u}_{-}\right)
$$

for all states $\boldsymbol{u} \in \mathcal{S}_{i}\left(\boldsymbol{u}_{-}\right)$between $\boldsymbol{u}_{-}$and $\boldsymbol{u}_{+}$. This condition generalizes the Oleinik entropy condition (52) for scalar equations to systems of conservation laws. In the particular case of three-phase flow, it is possible to arrive at a simpler condition. Because inflection loci are single connected curves, which correspond to maxima of eigenvalues, it can be shown [1] that condition (63) is equivalent to

$$
\nu_{i}\left(\boldsymbol{u}_{-}\right) \geq \sigma_{i}\left(\boldsymbol{u}_{+} ; \boldsymbol{u}_{-}\right)>\nu_{i}\left(\boldsymbol{u}_{+}\right) .
$$

Algorithms for calculating rarefaction-shock curves, based on a predictorcorrector strategy that achieves quadratic convergence, are given in Appendix A.3.

\subsubsection{Complete set of solutions}

Based on the analysis of the wave structure in Section 4.3.1, a wave of the $i$-family connecting two constant states may only be one of the following: an 
R. Juanes and T. W. Patzek: Analytical solution to the Riemann problem ... 31

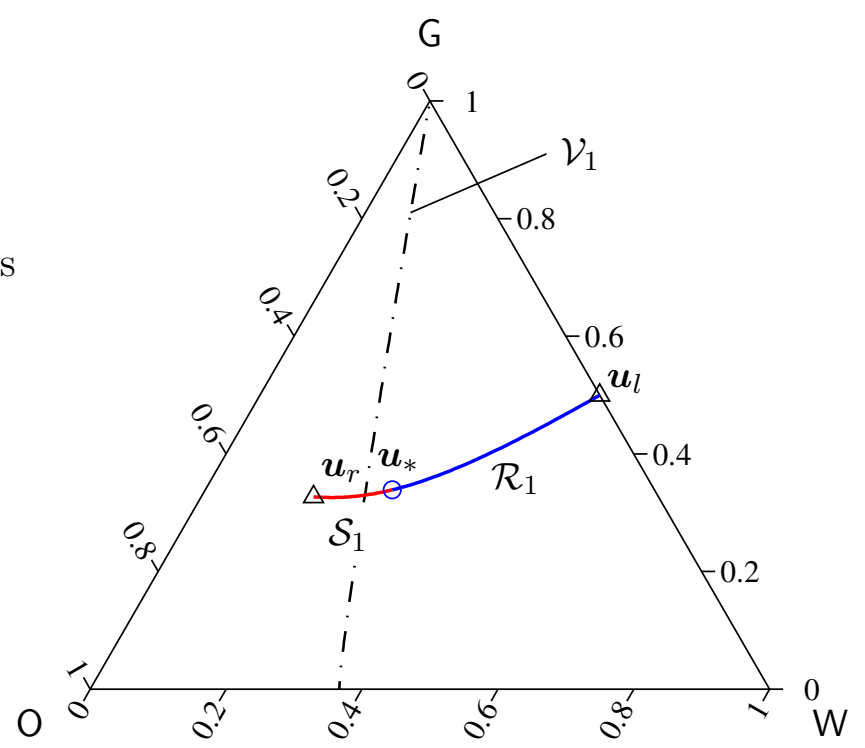

(a) Right state with $u_{r} \approx 0.17$

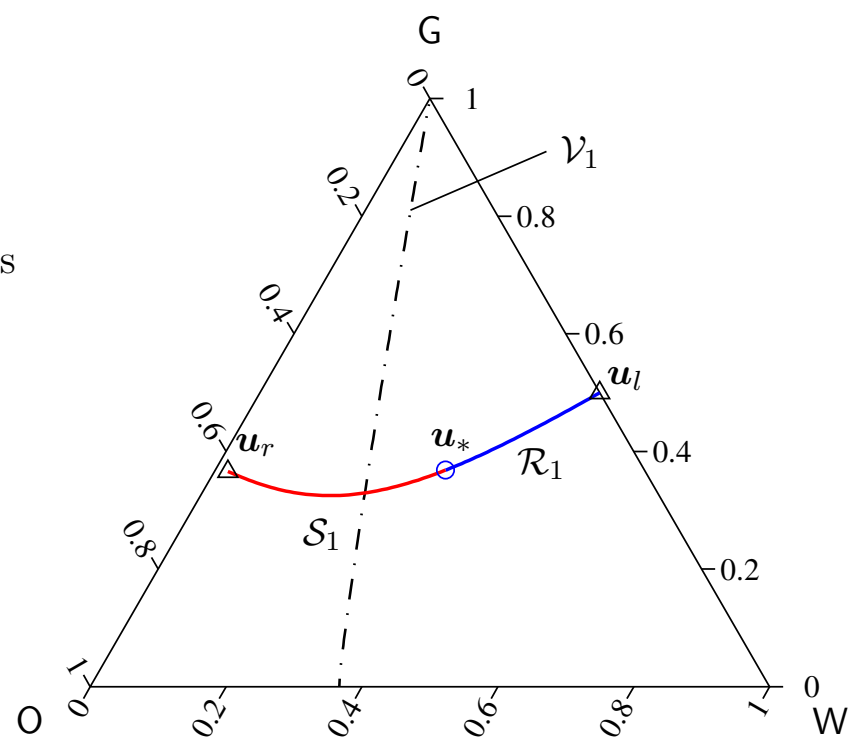

(b) Right state with $u_{r} \approx 0.02$

Figure 17. Rarefaction-shock curves of the 1-family with the same left state $\boldsymbol{u}_{l}=(0.5,0.5)$ and two different right states. The dash-dotted curve is the inflection locus of the 1-family. Note that the postshock state $\boldsymbol{u}_{*}$ is different for each case. 
R. Juanes and T. W. Patzek: Analytical solution to the Riemann problem . . 32

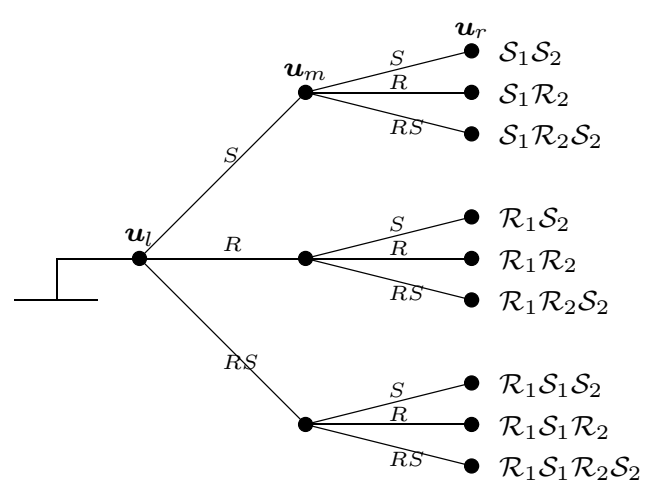

Figure 18. Schematic tree with all possible combinations of solutions to the Riemann problem of three-phase flow.

$i$-rarefaction $\left(\mathcal{R}_{i}\right)$, an $i$-shock $\left(\mathcal{S}_{i}\right)$, or an $i$-rarefaction-shock $\left(\mathcal{R}_{i} \mathcal{S}_{i}\right)$. Since the full solution to the Riemann problem is a sequence of two waves, $\mathcal{W}_{1}$ and $\mathcal{W}_{2}$, there are only 9 possible combinations of solutions. A schematic tree with all possible solution types is shown in Figure 18.

In Figure 19 we present the saturation paths in the ternary diagram for all 9 solution types. These are:

(a) $\mathcal{S}_{1} \mathcal{S}_{2}$ : both waves are genuine shocks and, therefore, the solution comprises three constant states separated by two discontinuities.

(b) $\mathcal{S}_{1} \mathcal{R}_{2}$ : the solution consists of a 1 -shock and a 2 -rarefaction.

(c) $\mathcal{S}_{1} \mathcal{R}_{2} \mathcal{S}_{2}$ : the solution comprises a genuine 1-shock through the left state and a composite 2-rarefaction-shock through the right state.

(d) $\mathcal{R}_{1} \mathcal{S}_{2}$ : the left state and the right state are joined by a 1-rarefaction followed by a 2 -shock.

(e) $\mathcal{R}_{1} \mathcal{R}_{2}$ : both waves are rarefactions, so the solution is continuous everywhere.

(f) $\mathcal{R}_{1} \mathcal{R}_{2} \mathcal{S}_{2}$ : a 1-rarefaction from the left state is followed by a composite 2 -rarefaction-shock to the right state.

(g) $\mathcal{R}_{1} \mathcal{S}_{1} \mathcal{S}_{2}$ : the slow wave emanating from the left state is a composite rarefaction-shock, which is followed by a genuine 2-shock to the right state. 
R. Juanes and T. W. Patzek: Analytical solution to the Riemann problem ... 33

(h) $\mathcal{R}_{1} \mathcal{S}_{1} \mathcal{R}_{2}$ : the left state is joined to the intermediate constant state by a composite rarefaction-shock, and the right state is reached along a 2-rarefaction.

(i) $\mathcal{R}_{1} \mathcal{S}_{1} \mathcal{R}_{2} \mathcal{S}_{2}$ : both waves are rarefaction-shocks.

All cases discussed above give the complete set of solutions to the Riemann problem of three-phase flow, when the following physical properties are satisfied: (1) the system is strictly hyperbolic; and (2) inflection loci are single connected curves, transversal to the integral curves, and correspond to maxima of the eigenvalues. Efficient algorithms for the complete calculation of the solution are given in Appendix B. They are based on a predictor-corrector strategy coupled with a full Newton iteration, which achieves quadratic convergence in all cases.

\section{Application example: water-gas injection}

In this section we describe in some detail a synthetic example, where we apply the analytical solution of three-phase flow presented in Section 4. The objective is twofold:

1. Illustrate the applicability and potential of the theory to develop exact solutions for problems of great practical interest.

2. Show that the wave structure arising in three-phase flow displacements should not be approximated by a sequence of two consecutive two-phase flow displacements.

\subsection{Description of the problem}

The problem involves simultaneous injection of water and gas into a core that is initially filled with oil and water, as shown in Figure 20. Initially, the core has constant reduced (normalized) saturations of 0.95 oil saturation, and 0.05 water saturation. Gas and water are injected in such proportion that the reduced water and gas saturations at the inlet are 0.5 and 0.5 , respectively. The injected reduced saturations are assumed to be constant throughout the experiment.

This physical problem is modeled mathematically as a Riemann problem, where two initially constant states are separated by a single discontinuity. 
R. Juanes and T. W. Patzek: Analytical solution to the Riemann problem ... 34
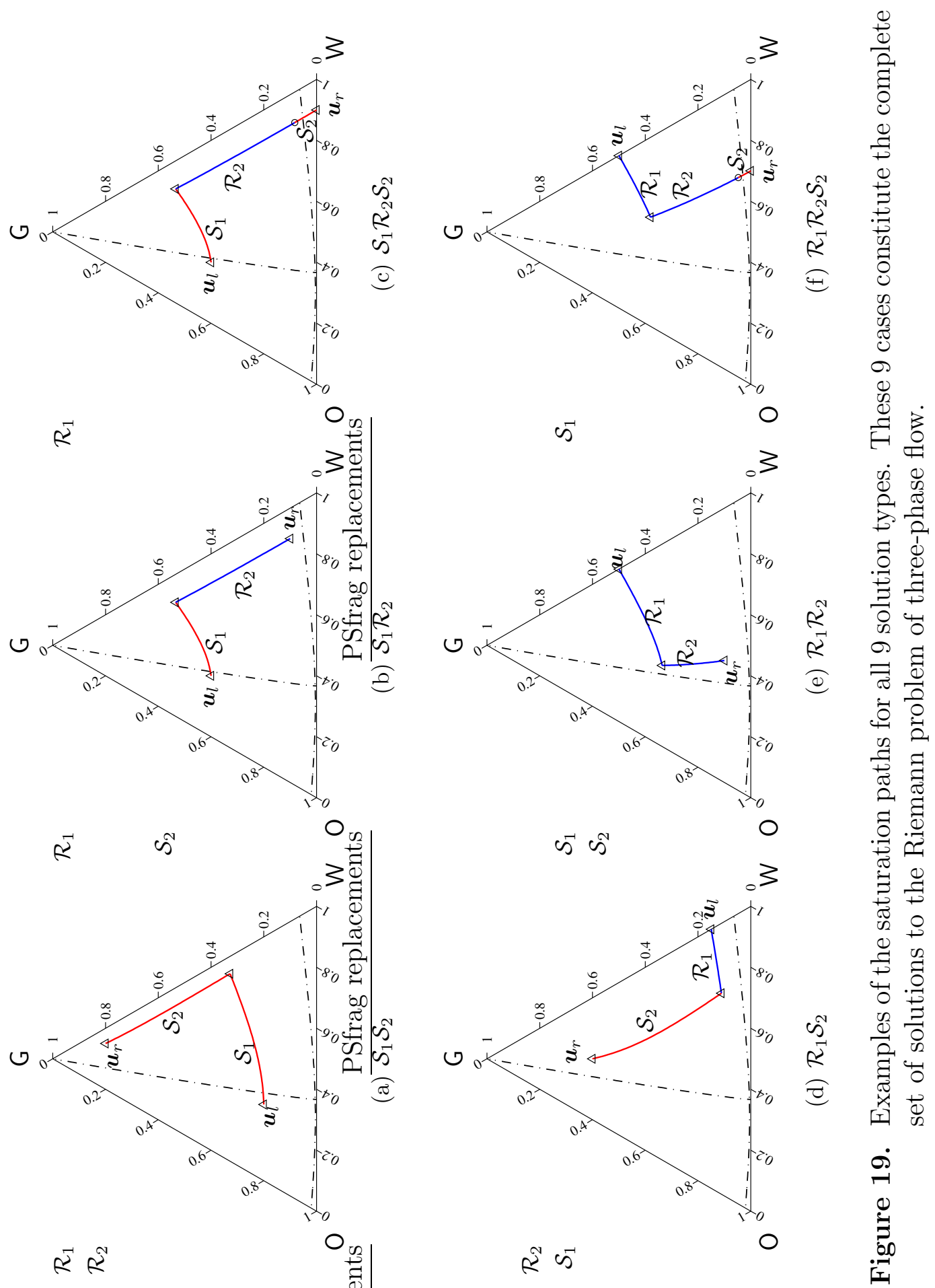
R. Juanes and T. W. Patzek: Analytical solution to the Riemann problem ... 35

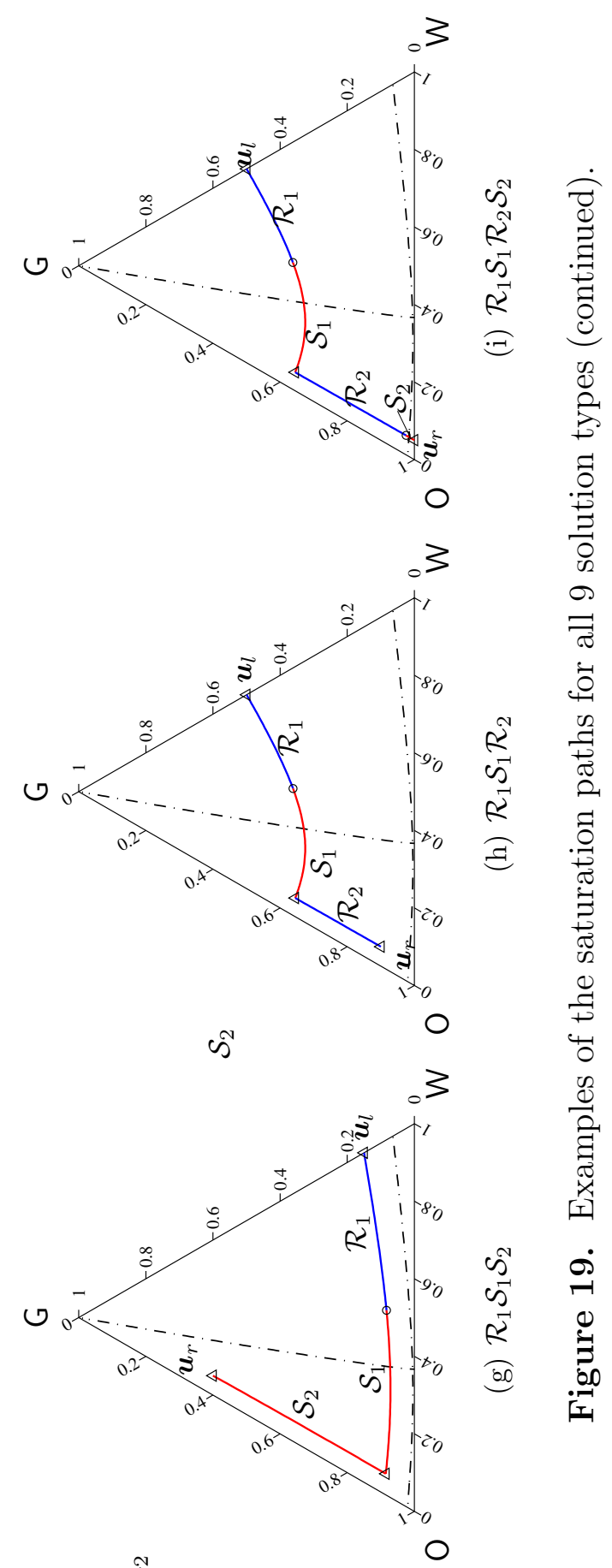


R. Juanes and T. W. Patzek: Analytical solution to the Riemann problem ... 36

Injected saturations Initial saturations

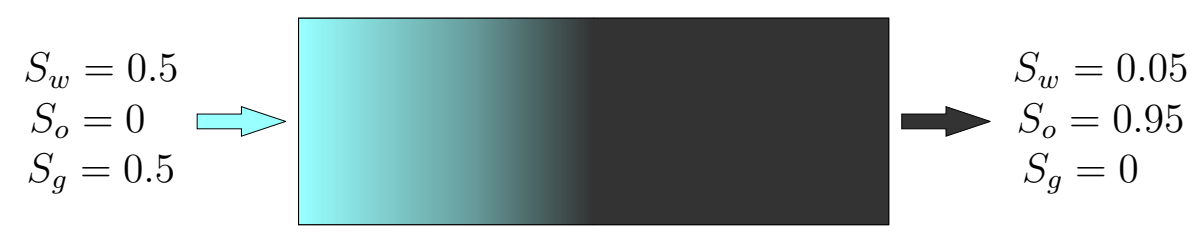

Figure 20. Sketch of the injection problem. Water and gas are injected into a core initially filled with oil and water.

Here, the left (injected) saturation state is $\boldsymbol{u}_{l}=(0.5,0.5)$, and the right (initial) saturation state is $\boldsymbol{u}_{r}=(0.05,0)$. We use the relative mobilities in Equations (30)-(32) with $\beta_{g}=0.1$, and the fluid viscosities in (35).

\subsection{Exact solution}

The exact solution turns out to be of type $\mathcal{R}_{1} \mathcal{S}_{1} \mathcal{R}_{2} \mathcal{S}_{2}$, that is, both waves are rarefaction-shocks: $\mathcal{W}_{1} \equiv \mathcal{R}_{1} \mathcal{S}_{1}$, and $\mathcal{W}_{2} \equiv \mathcal{R}_{2} \mathcal{S}_{2}$. The variables that need to be determined to fully characterize the solution are: the intermediate constant state $\boldsymbol{u}_{m}$, the shock speeds $\sigma_{1}$ and $\sigma_{2}$, and the post-shock states $\boldsymbol{u}_{1}^{*}$ and $\boldsymbol{u}_{2}^{*}$ of each wave. The constant state $\boldsymbol{u}_{m}$ corresponds to the intersection of the two wave curves, while the post-shock states are the points where the rarefaction curve and the shock curve of the same family are joined. Schematically, this can be represented as follows:

$$
\boldsymbol{u}_{l} \stackrel{\mathcal{R}_{1}}{\longrightarrow} \boldsymbol{u}_{1}^{*} \stackrel{\mathcal{S}_{1}}{\longrightarrow} \boldsymbol{u}_{m} \stackrel{\mathcal{R}_{2}}{\longrightarrow} \boldsymbol{u}_{2}^{*} \stackrel{\mathcal{S}_{2}}{\longrightarrow} \boldsymbol{u}_{r}
$$

A detailed algorithm to obtain this solution is given in Appendix B.3.

In Figure 21 we show the saturation path of the exact solution. The corresponding saturation profiles are plotted in Figure 22 against the similarity variable $\zeta=x / t$. Of course, the right state coincides with the initial saturations ( $95 \%$ oil and $5 \%$ water) and the left state is given by the injected saturations ( $50 \%$ water and $50 \%$ gas). Because the characteristic speeds of the slow and fast waves are very different, the entire saturation profile shown on the right plot $22(\mathrm{~b})$ does not allow to visualize the structure of the slow wave (1-wave). A detail of the 1 -wave is shown on the left plot 22 (a). It is apparent that the 1-wave involves changes in the saturation of all three fluids. 


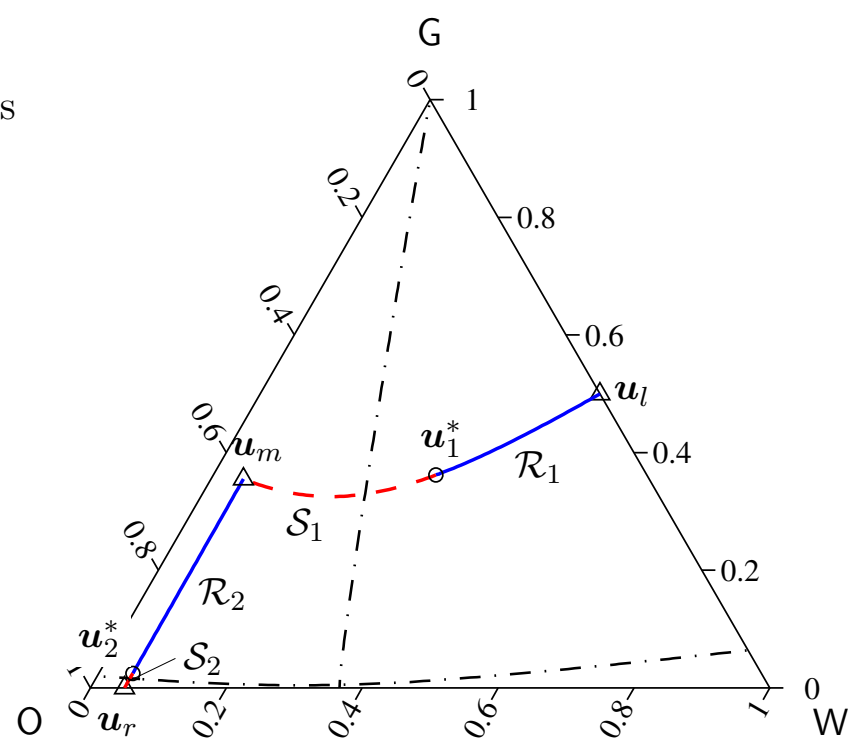

Figure 21. Saturation path of the exact solution to the water-gas injection problem. Both waves are rarefaction-shocks $\left(\mathcal{R}_{1} \mathcal{S}_{1} \mathcal{R}_{2} \mathcal{S}_{2}\right.$ solution). Dash-dotted curves represent the inflection loci.

\subsection{Approximate solution}

Classical analytical solutions of three-phase flow are usually restricted to the case when saturation paths are straight lines $[17,24,38,46]$. This approximation dates back to the early conceptual model of a waterflood in the presence of gas by Kyte et al. [23]. In general, straight saturation paths arise only when the relative permeability of each phase is assumed to be a linear function of its own saturation.

The physical motivation for assuming saturation paths that are straight lines parallel to the edges of the ternary diagram is to split the actual threephase flow displacement into a sequence of two successive two-phase displacements. In the context of the water-gas injection problem described above, this approximation is equivalent to assuming that the fast wave is a displacement of oil exclusively by gas, and that the slow wave is a displacement of oil exclusively by water. Therefore, it is assumed that the water saturation is constant along the fast wave, and the gas saturation is constant along the slow wave. The immediate benefit of this simplification is that the solution may be computed using the theory of two-phase Buckley-Leverett flow. Here 
R. Juanes and T. W. Patzek: Analytical solution to the Riemann problem ... 38

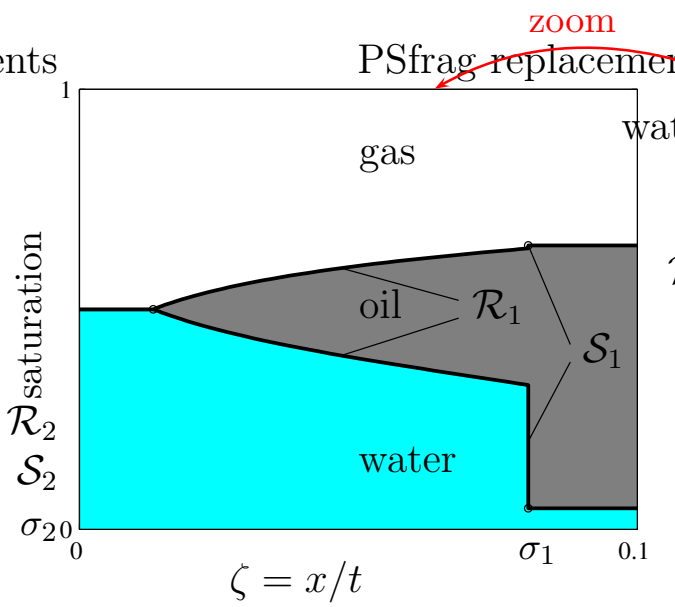

(a) Detail of the 1-wave

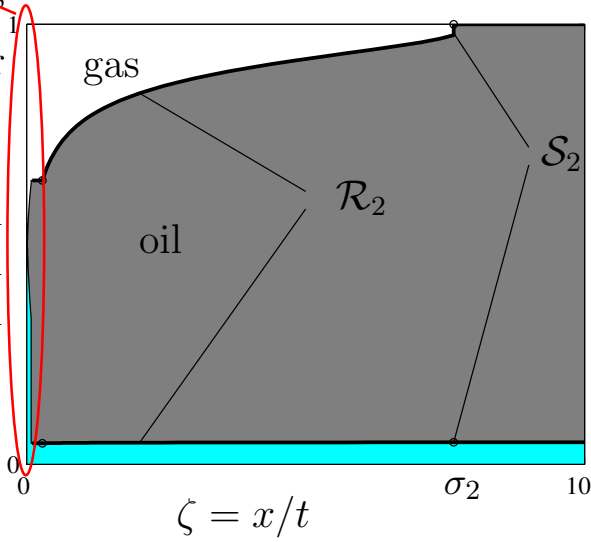

(b) Entire saturation profile

Figure 22. Saturation profiles of the exact solution to the water-gas injection problem. Saturations of each phase are plotted against the similarity variable $\zeta=x / t$. The right plot (b) shows the entire saturation profile. Because of the very different characteristic speeds of the slow and fast waves, we show a detail of the 1-wave on the left plot (a).

we evaluate the accuracy of this simplifying assumption.

In Figure 23 we show the saturation paths that result from the assumption described above. The intermediate constant state $\boldsymbol{u}_{m}$ is obvious to calculate as the intersection of the two wave paths. Each wave is then resolved using the catalogue of two-phase flow solutions in Section 4.2. It turns out that the slow wave is a 1 -shock $\left(\mathcal{W}_{1} \equiv \mathcal{S}_{1}\right)$, and the fast wave is a 2-rarefaction-shock $\left(\mathcal{W}_{2} \equiv \mathcal{R}_{2} \mathcal{S}_{2}\right)$. Thus, the wave structure is different from that of the exact solution. In Figure 24 we plot the saturation profiles of the approximate solution against the similarity variable $\zeta$. The right figure 24(b) shows the entire saturation profile, and the left figure 24(a) a detail of the 1-wave. It is evident that, while the qualitative behavior of the fast wave is similar to that of the exact solution (see Figure 22(b)), the structure of the slow wave is very different (compare with Figure 22(a)). 
R. Juanes and T. W. Patzek: Analytical solution to the Riemann problem . . 39

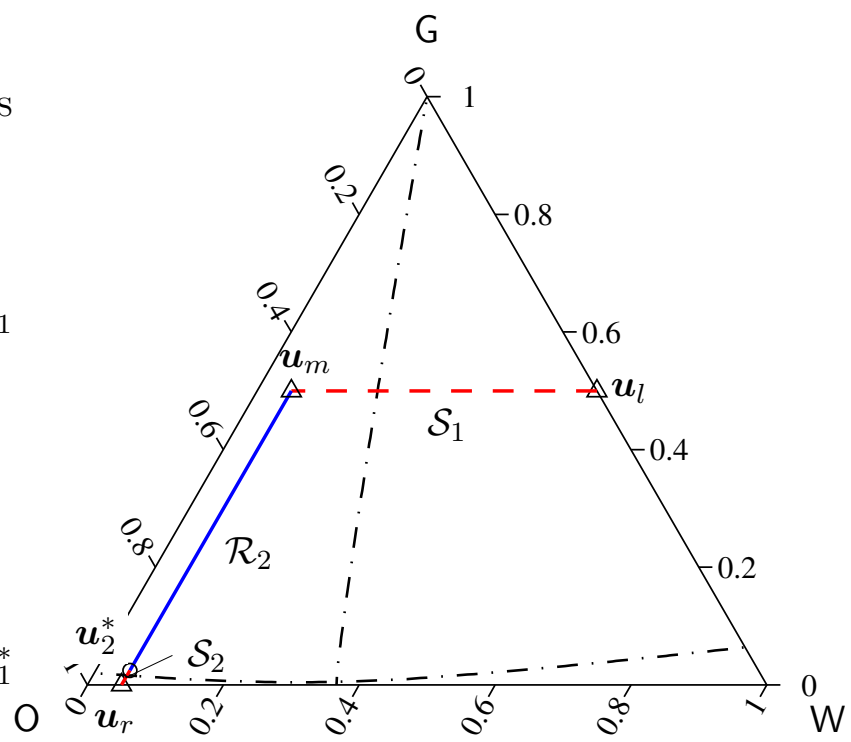

Figure 23. Saturation path of the approximate solution to the water-gas injection problem, which assumes that saturation paths are straight lines, parallel to the edges of the ternary diagram. The slow wave is a 1-shock, and the fast wave is a 2-rarefaction-shock $\left(\mathcal{S}_{1} \mathcal{R}_{2} \mathcal{S}_{2}\right.$ solution $)$. Each individual wave is fully determined using the theory of two-phase displacements.

\subsection{Discussion}

To better evaluate the accuracy of the straight-line approximation of the saturation paths, we compare the oil production of the exact and the approximate solutions. This is done by taking a fixed length $L$ of the core, and calculating the amount of oil displaced at the outlet at any given time. Using the definitions of dimensionless space and time coordinates in Equations (11)-(12), and the similarity variable in Equation (41), we plot the results against the dimensionless time $\tau:=1 / \zeta=t / x$. The variables of interest are:

1. Oil production rate. This quantity is proportional to the oil fractional flow $f_{\text {oil }}$ at the outlet face, that is:

$$
f_{\text {oil }}(\tau)=1-f(\tau)-g(\tau)
$$


R. Juanes and T. W. Patzek: Analytical solution to the Riemann problem ... 40

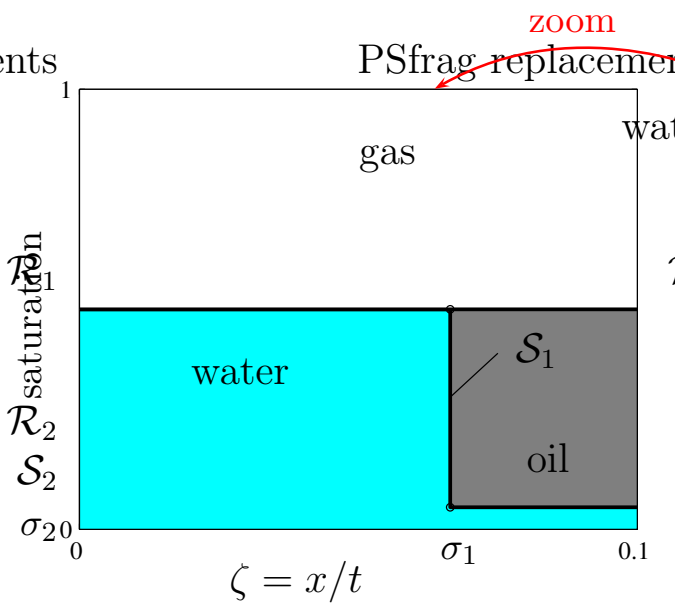

(a) Detail of the 1-wave

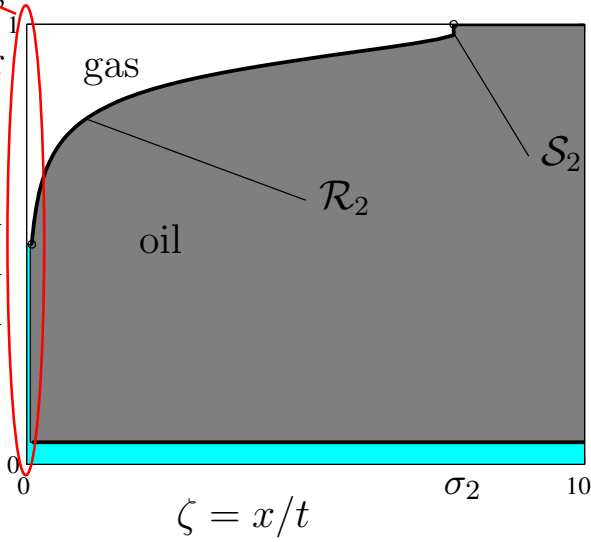

(b) Entire saturation profile

Figure 24. Saturation profiles of the approximate solution to the watergas injection problem, which assumes that the three-phase flow displacement is split into a sequence of two successive two-phase displacements. Saturations of each phase are plotted against the similarity variable $\zeta=x / t$. The right plot (b) shows the entire saturation profile. The left plot (a) shows a detail of the 1-wave.

2. Cumulative oil production. This quantity is proportional to the fraction $Q_{\text {oil }}$ of the original oil in place that has been swept through the outlet face, that is:

$$
Q_{\text {oil }}(\tau)=\int_{0}^{\tau} f_{\text {oil }}(\eta) \mathrm{d} \eta
$$

In Figure 25 we plot the dimensionless oil production rate $f_{\text {oil }}$ defined in Equation (66), as predicted by the exact solution and the approximate solution. The approximate solution agrees well with the exact solution at early times (roughly, for $\tau<3$ ). The reason for this good agreement is that, at early times, only the fast wave has reached the outlet face, and the exact saturation path of the fast wave may be approximated accurately by a straight line of constant water saturation (compare Figure 23 with 21, and Figure 24(b) with 22(b)). However, the approximate solution deviates very significantly from the exact solution for times $\tau>3$, because both the saturation and the speed of propagation of the oil bank are predicted incorrectly. 
R. Juanes and T. W. Patzek: Analytical solution to the Riemann problem ... 41

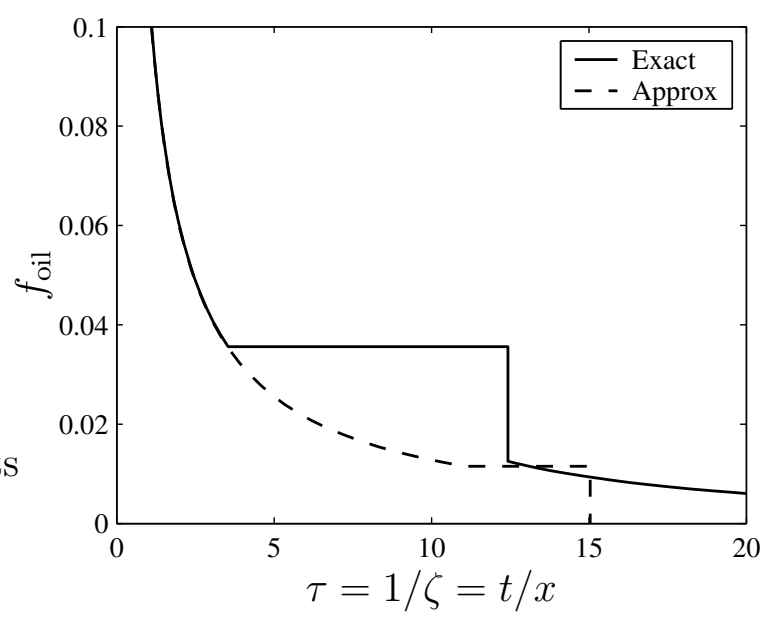

Figure 25. Comparison of the dimensionless oil production rate predicted by the exact solution and the approximate solution. The approximate solution deviates very significantly from the exact solution because both the saturation and the speed of propagation of the oil bank are incorrect.

A faulty behavior of the approximate solution is better visualized by plotting the dimensionless cumulative oil production $Q_{\text {oil }}$ defined in Equation (67), which is simply the area under the curve in Figure 25. This quantity is shown in Figure 26 for both the exact and the approximate solutions. The curve given by the exact solution tends asymptotically to a value of $Q_{\text {oil,max }}=0.95$, which is precisely the initial reduced oil saturation. This is required for mass conservation. In contrast, the curve predicted by the approximate solution reaches a plateau of $Q_{\text {oil, } \max } \approx 0.67$ at time $\tau \approx 15$. This behavior illustrates that the approximate solution is not mass conservative.

The results presented above motivate the following remarks:

1. Rarefaction-shocks waves, common in two-phase displacements, appear also in realistic scenarios of three-phase flow.

2. In the realm of Buckley-Leverett models of three-phase flow, individual waves involve simultaneous three-phase displacements.

3. In general, the saturation paths induced by the exact characteristic waves are not straight lines on the ternary diagram. Saturation paths 
R. Juanes and T. W. Patzek: Analytical solution to the Riemann problem ... 42

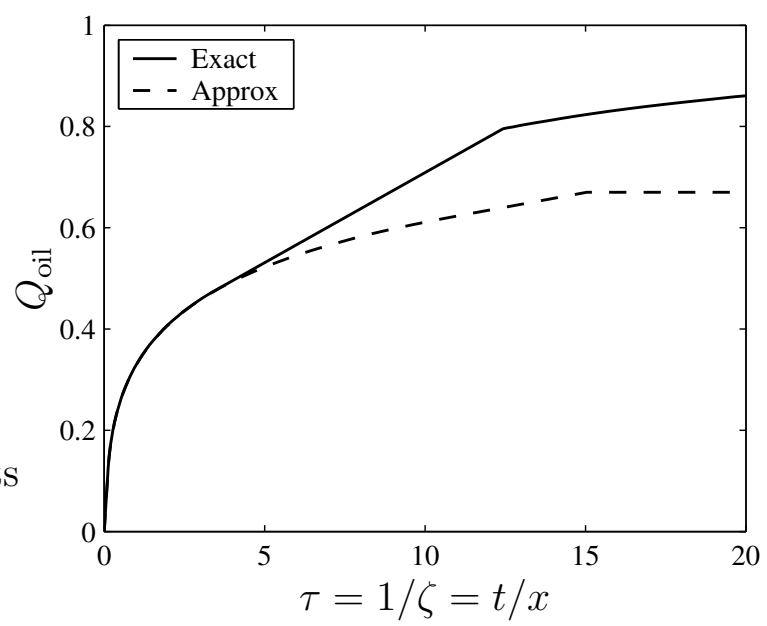

Figure 26. Comparison of the dimensionless cumulative oil production predicted by the exact solution and the approximate solution. The exact solution tends asymptotically to a value of 0.95 , consistent with the original reduced oil saturation. The approximate solution does not, which indicates it does not satisfy the overall mass balance.

are straight lines only for linear relative permeability functions, or for very particular initial and injected saturation states.

4. Splitting a three-phase displacement into a sequence of two successive two-phase displacements - for which saturation paths are straight lines parallel to the edges of the ternary diagram - is an assumption that may not be appropriate.

5. In particular, it was shown by means of a representative example that the approximate solution calculated under this assumption does not satisfy overall mass balance, and leads to very inaccurate predictions of oil recovery.

\section{Conclusions}

The key result of this paper is the complete general analytical solution to the Riemann problem of three-phase flow of immiscible, incompressible fluids, 
R. Juanes and T. W. Patzek: Analytical solution to the Riemann problem ... 43

when gravity and capillarity are not included in the formulation. The solution comprises two waves, a (slow) 1-wave and a (fast) 2-wave, separated by an intermediate constant saturation state. Each of these two waves may only be a rarefaction, a shock, or a rarefaction-shock. Thus, there can only be 9 possible combinations of admissible waves. All these combinations, which constitute the complete set of solutions to the Riemann problem, are discussed in our paper. We demonstrate that, in general, a three-phase flow displacement should not be approximated by a sequence of two successive two-phase displacements. Such approximation does not satisfy an overall mass balance, and may lead to very inaccurate predictions of oil recovery.

We are currently extending the analytical solution presented here to the case when saturation states are outside the three-phase flow region, that is, when one or more phases are immobile $[13,14]$. Capillarity effects, which smear the traveling discontinuities of the capillarity-free solution, may also be incorporated through the method of asymptotic expansions [6].

We anticipate that the results of this paper will be relevant to: (1) the interpretation of three-phase displacement experiments; (2) implementation of the solution in streamtube simulators; (3) development of improved relative permeability models; and (4) validation of numerical methods.

\section{ACKNOWLEDGEMENTS}

The authors wish to express their gratitude to Dr. Dmitriy B. Silin for his careful revision of the manuscript. This work was supported by the Laboratory Directed Research and Development Program of Lawrence Berkeley National Laboratory under the Department of Energy Contract No. DE-AC03-76SF00098. Funding provided by Barrié de la Maza, Jane Lewis, and Repsol-YPF fellowships, awarded to the first author, is also gratefully acknowledged.

\section{References}

[1] F. Ancona and A. Marson. A note on the Riemann problem for general $n \times n$ conservation laws. J. Math. Anal. Appl., 260:279-293, 2001.

[2] S. Axler. Linear Algebra Done Right. Undergraduate Texts in Mathematics. Springer-Verlag, New York, 1996. 
R. Juanes and T. W. Patzek: Analytical solution to the Riemann problem ... 44

[3] K. Aziz and A. Settari. Petroleum Reservoir Simulation. Elsevier, London, 1979.

[4] L. E. Baker. Three-phase relative permeability correlations. In SPE/DOE Sixth Symposium on Enhanced Oil Recovery, Tulsa, OK, April 17-20, 1988. (SPE/DOE 17369).

[5] G. I. Barenblatt. Scaling, Self-Similarity, and Intermediate Asymptotics. Cambridge Texts in Applied Mathematics. Cambridge University Press, 1996.

[6] G. I. Barenblatt, V. M. Entov, and V. M. Ryzhik. Theory of Fluid Flows through Natural Rocks, volume 3 of Theory and Applications of Transport in Porous Media. Kluwer, Dordrecht, 1990. Expanded and revised edition of the original in Russian Dvizhenie zhidkostei i gazov $v$ prirodnykh plastakh, Nedra Publishers, 1984.

[7] J. Bear. Dynamics of Fluids in Porous Media. Environmental Science Series. Elsevier, New York, 1972. Reprinted with corrections, Dover, New York, 1988.

[8] J. B. Bell, J. A. Trangenstein, and G. R. Shubin. Conservation laws of mixed type describing three-phase flow in porous media. SIAM J. Appl. Math., 46(6):1000-1017, 1986.

[9] S. E. Buckley and M. C. Leverett. Mechanism of fluid displacement in sands. Petrol. Trans. AIME, 146:107-116, 1942.

[10] G. Chavent and J. Jaffré. Mathematical Models and Finite Elements for Reservoir Simulation, volume 17 of Studies in Mathematics and its Applications. Elsevier, North-Holland, 1986.

[11] G. Chavent, J. Jaffré, and S. Jan-Jégou. Estimation of relative permeabilities in three-phase flow in porous media. Inverse Problems, 15:3339, 1999.

[12] C. M. Dafermos. Hyperbolic Conservation Laws in Continuum Physics, volume 325 of A Series of Comprehensive Studies in Mathematics. Springer-Verlag, Berlin, 2000. 
R. Juanes and T. W. Patzek: Analytical solution to the Riemann problem ... 45

[13] A. H. Falls and W. M. Schulte. Features of three component, three phase displacement in porous media. SPERE, 7(4):426-432, November 1992.

[14] A. H. Falls and W. M. Schulte. Theory of three component, three phase displacement in porous media. SPERE, 7(3):377-384, August 1992.

[15] F. J. Fayers. Extension of Stone's method I and conditions for real characteristics in three-phase flow. In SPE 62nd Annual Technical Conference and Exhibition, Dallas, TX, September 27-30, 1987. (SPE 16965).

[16] S. K. Godunov. A difference scheme for numerical computation of discontinuous solutions of equations of fluid dynamics (in Russian). Mat. Sb., 47(89):271-306, 1959.

[17] F. G. Helfferich. Theory of multicomponent, multiphase displacement in porous media. SPEJ, 21(1):51-62, February 1981. Petrol. Trans. AIME, 271.

[18] L. Holden. On the strict hyperbolicity of the Buckley-Leverett equations for three-phase flow in a porous medium. SIAM J. Appl. Math., 50(3):667-682, 1990.

[19] P. J. Hicks Jr. and A. S. Grader. Simulation of three-phase displacement experiments. Transp. Porous Media, 24:221-245, 1996.

[20] R. Juanes and T. W. Patzek. Relative permeabilities for strictly hyperbolic models of three-phase flow in porous media. Transp. Porous Media, 2002. (Submitted).

[21] R. Juanes, D. B. Silin, and T. W. Patzek. On elliptic regions in models of three-phase flow through permeable rocks. (In preparation).

[22] B. L. Keyfitz and M. Shearer, editors. Nonlinear Evolution Equations that Change Type, volume 27 of The IMA Volumes in Mathematics and its Applications, New York, 1990. Springer-Verlag.

[23] J. R. Kyte, R. J. Stanclift Jr., S. C. Stephan Jr., and L. A. Rapoport. Mechanism of water flooding in the presence of free gas. Petrol. Trans. AIME, 207:215-221, 1956.

[24] L. W. Lake. Enhanced Oil Recovery. Prentice-Hall, Englewood Cliffs, NJ, 1989. 
R. Juanes and T. W. Patzek: Analytical solution to the Riemann problem ... 46

[25] P. D. Lax. Hyperbolic systems of conservation laws, II. Comm. Pure Appl. Math., 10:537-566, 1957.

[26] R. J. LeVeque. Numerical Methods for Conservation Laws. Birkhäuser Verlag, Berlin, second edition, 1992.

[27] W. B. Lindquist, editor. Current Progress in Hyperbolic Systems: Riemann Problems and Computations, volume 100 of Contemporary Mathematics, Providence, RI, 1989. American Mathematical Society.

[28] T.-P. Liu. The Riemann problem for general $2 \times 2$ conservation laws. Trans. Amer. Math. Soc., 199:89-112, 1974.

[29] T.-P. Liu. The Riemann problem for general systems of conservation laws. J. Differential Equations, 18:218-234, 1975.

[30] D. Marchesin and H. B. Medeiros. A note on the stability of eigenvalue degeneracy in nonlinear conservation laws of multiphase flow. In W. B. Lindquist, editor, Current Progress in Hyperbolic Systems: Riemann Problems and Computations, volume 100 of Contemporary Mathematics, pages 215-224. American Mathematical Society, Providence, RI, 1989.

[31] D. Marchesin and B. J. Plohr. Wave structure in WAG recovery. SPEJ, 6(2):209-219, June 2001.

[32] C. T. Miller, G. Christakos, P. T. Imhoff, J. F. McBride, J. A. Pedit, and J. A. Trangenstein. Multiphase flow and transport modeling in heterogeneous porous media: challenges and approaches. Adv. Water Resour., 21(2):77-120, 1998.

[33] M. Muskat. Physical Principles of Oil Production. McGraw-Hill, New York, 1949.

[34] M. J. Oak, L. E. Baker, and D. C. Thomas. Three-phase relative permeability of Berea sandstone. JPT, 42(8):1054-1061, August 1990.

[35] O. A. Oleinik. Discontinuous solutions of nonlinear differential equations. Usp. Mat. Nauk. (N.S.), 12:3-73, 1957. English transl. in Amer. Math. Soc. Transl. Ser. 2, 26:95-172. 
R. Juanes and T. W. Patzek: Analytical solution to the Riemann problem ... 47

[36] J. C. Parker, R. J. Lenhard, and T. Kuppusamy. A parametric model for constitutive properties governing multiphase flow in porous media. Water Resour. Res., 23(4):618-624, 1987.

[37] D. W. Peaceman. Fundamentals of Numerical Reservoir Simulation, volume 6 of Developments in Petroleum Science. Elsevier, Amsterdam, 1977.

[38] G. A. Pope. The application of fractional flow theory to enhanced oil recovery. SPEJ, 20(3):191-205, June 1980. Petrol. Trans. AIME, 269.

[39] M. Shearer. Loss of strict hyperbolicity of the Buckley-Leverett equations for three phase flow in a porous medium. In M. F. Wheeler, editor, Numerical Simulation in Oil Recovery, volume 11 of The IMA Volumes in Mathematics and its Applications, pages 263-283. Springer-Verlag, New York, 1988.

[40] M. Shearer and J. A. Trangenstein. Loss of real characteristics for models of three-phase flow in a porous medium. Transp. Porous Media, 4:499$525,1989$.

[41] J. Smoller. Shock Waves and Reaction-Diffusion Equations, volume 258 of A Series of Comprehensive Studies in Mathematics. Springer-Verlag, New York, second edition, 1994.

[42] H. L. Stone. Probability model for estimating three-phase relative permeability. JPT, 23(2):214-218, February 1970. Petrol. Trans. AIME, 249.

[43] H. L. Stone. Estimation of three-phase relative permeability and residual oil data. J. Can. Petrol. Technol., 12(4):53-61, 1973.

[44] J. A. Trangenstein. Three-phase flow with gravity. In W. B. Lindquist, editor, Current Progress in Hyperbolic Systems: Riemann Problems and Computations, volume 100 of Contemporary Mathematics, pages 147159. American Mathematical Society, Providence, RI, 1989.

[45] H. J. Welge. A simplified method for computing oil recovery by gas or water drive. Petrol. Trans. AIME, 195:91-98, 1952. 
R. Juanes and T. W. Patzek: Analytical solution to the Riemann problem ... 48

[46] G. P. Willhite. Waterflooding, volume 3 of SPE Textbook Series. Society of Petroleum Engineers, Richardson, TX, 1986.

[47] E. Zauderer. Partial Differential Equations of Applied Mathematics. Series in Pure and Applied Mathematics. John Wiley \& Sons, New York, 1983.

\section{A Solution algorithms for the wave curves of three-phase flow}

\section{A.1 Rarefaction curves}

Rarefaction curves are subsets of the integral curves, satisfying that the eigenvalue increases monotonically along the curve. Let $\tilde{\boldsymbol{u}}_{i}(\xi)=\left(\tilde{u}_{i}(\xi), \tilde{v}_{i}(\xi)\right)$ be a parameterization of a rarefaction curve of the $i$-family, $\mathcal{R}_{i}(\hat{\boldsymbol{u}})$, starting at the reference state $\hat{\boldsymbol{u}}=(\hat{u}, \hat{v})$. From the definition of a rarefaction curve, it satisfies the initial value problem:

$$
\left\{\begin{array}{l}
\tilde{\boldsymbol{u}}_{i}^{\prime}(\xi)=\alpha(\xi) \boldsymbol{r}_{i}\left(\tilde{\boldsymbol{u}}_{i}(\xi)\right), \\
\tilde{\boldsymbol{u}}_{i}(0)=\hat{\boldsymbol{u}}
\end{array}\right.
$$

where $\alpha(\xi)$ is some scalar factor.

For a 1-rarefaction curve we choose the following parameterization:

$$
\tilde{u}_{1}(\xi)=\hat{u}+\xi .
$$

Using Equation (28), the initial value problem (68) reduces to

$$
\left\{\begin{array}{l}
\tilde{v}_{1}^{\prime}(\xi)=\frac{r_{1 v}}{r_{1 u}}=\frac{\nu_{1}-f_{, u}}{f_{, v}}=\frac{g_{, u}}{\nu_{1}-g_{, v}}, \\
\tilde{v}_{1}(0)=\hat{v}
\end{array}\right.
$$

For a 2-rarefaction curve we use the parameterization

$$
\tilde{v}_{2}(\xi)=\hat{v}+\xi
$$

Using Equation (29), the initial value problem (68) takes the form

$$
\left\{\begin{array}{l}
\tilde{u}_{2}^{\prime}(\xi)=\frac{r_{2 u}}{r_{2 v}}=\frac{f_{, v}}{\nu_{2}-f_{, u}}=\frac{\nu_{2}-g_{, v}}{g_{, u}} \\
\tilde{u}_{2}(0)=\hat{u}
\end{array}\right.
$$


R. Juanes and T. W. Patzek: Analytical solution to the Riemann problem ... 49

A simple Runge-Kutta algorithm can be used to integrate the scalar ordinary differential equations (70) and (72).

\section{A.2 Shock curves}

Shock curves are subsets of the Hugoniot loci, satisfying the Lax entropy condition. Here we describe a Newton iterative procedure for the calculation of the shock curves. Let $\tilde{\boldsymbol{u}}_{i}(\xi)=\left(\tilde{u}_{i}(\xi), \tilde{v}_{i}(\xi)\right)$ be a parameterization of an $i$-shock curve, $\mathcal{S}_{i}(\hat{\boldsymbol{u}})$, starting at the reference state $\hat{\boldsymbol{u}}=(\hat{u}, \hat{v})$.

We use the following parameterization of the 1-shock curve:

$$
\tilde{u}_{1}(\xi)=\hat{u}+\xi
$$

For each value of the parameter $\xi, \tilde{u}_{1}$ is known from (73), and the following system of algebraic equations needs to be solved for $\sigma_{1}$ and $\tilde{v}_{1}$ :

$$
\begin{aligned}
& R_{1}\left(\sigma_{1}, \tilde{v}_{1}\right):=f\left(\tilde{u}_{1}, \tilde{v}_{1}\right)-f(\hat{u}, \hat{v})-\sigma_{1}\left(\tilde{u}_{1}-\hat{u}\right)=0 \\
& R_{2}\left(\sigma_{1}, \tilde{v}_{1}\right):=g\left(\tilde{u}_{1}, \tilde{v}_{1}\right)-g(\hat{u}, \hat{v})-\sigma_{1}\left(\tilde{v}_{1}-\hat{v}\right)=0 .
\end{aligned}
$$

We define the solution vector $\boldsymbol{x}$ and the residual vector $\boldsymbol{R}$ as:

$$
\boldsymbol{x}:=\left(\begin{array}{c}
\sigma_{1} \\
\tilde{v}_{1}
\end{array}\right), \quad \boldsymbol{R}:=\left(\begin{array}{c}
R_{1} \\
R_{2}
\end{array}\right) .
$$

We use Newton's method to find the solution to the system (74), as indicated in Figure 27.

For the 2-shock curve we use the following parameterization:

$$
\tilde{v}_{2}(\xi)=\hat{v}+\xi
$$

The iterative procedure to obtain the solution is identical to that of the 1shock curve, but now the system needs to be solved for $\tilde{u}_{2}$ and $\sigma_{2}$. The solution vector, residual vector and Jacobian matrix are:

$$
\begin{aligned}
\boldsymbol{x} & =\left(\begin{array}{c}
\tilde{u}_{2} \\
\sigma_{2}
\end{array}\right), \\
\boldsymbol{R} & =\left(\begin{array}{c}
f\left(\tilde{u}_{2}, \tilde{v}_{2}\right)-f(\hat{u}, \hat{v})-\sigma_{2}\left(\tilde{u}_{2}-\hat{u}\right) \\
g\left(\tilde{u}_{2}, \tilde{v}_{2}\right)-g(\hat{u}, \hat{v})-\sigma_{2}\left(\tilde{v}_{2}-\hat{v}\right)
\end{array}\right), \\
\boldsymbol{J} & =\frac{\partial \boldsymbol{R}}{\partial \boldsymbol{x}}=\left(\begin{array}{cc}
f_{, u}\left(\tilde{u}_{2}, \tilde{v}_{2}\right)-\sigma_{2} & -\left(\tilde{u}_{2}-\hat{u}\right) \\
g_{, u}\left(\tilde{u}_{2}, \tilde{v}_{2}\right) & -\left(\tilde{v}_{2}-\hat{v}\right)
\end{array}\right) .
\end{aligned}
$$


R. Juanes and T. W. Patzek: Analytical solution to the Riemann problem ... 50

1. Set $k=0$. Initial guess:

$$
\boldsymbol{x}^{(0)}=\left(\begin{array}{c}
\sigma_{1}^{(0)} \\
\tilde{v}_{1}^{(0)}
\end{array}\right)
$$

2. Evaluate residual vector and Jacobian matrix:

$$
\begin{aligned}
& \boldsymbol{R}^{(k)}=\left(\begin{array}{c}
f\left(\tilde{u}_{1}, \tilde{v}_{1}^{(k)}\right)-f(\hat{u}, \hat{v})-\sigma_{1}^{(k)}\left(\tilde{u}_{1}-\hat{u}\right) \\
g\left(\tilde{u}_{1}, \tilde{v}_{1}^{(k)}\right)-g(\hat{u}, \hat{v})-\sigma_{1}^{(k)}\left(\tilde{v}_{1}^{(k)}-\hat{v}\right)
\end{array}\right), \\
& \boldsymbol{J}^{(k)}=\left.\frac{\partial \boldsymbol{R}}{\partial \boldsymbol{x}}\right|^{(k)}=\left(\begin{array}{cc}
-\left(\tilde{u}_{1}-\hat{u}\right) & f_{, v}\left(\tilde{u}_{1}, \tilde{v}_{1}^{(k)}\right) \\
-\left(\tilde{v}_{1}^{(k)}-\hat{v}\right) & g_{, v}\left(\tilde{u}_{1}, \tilde{v}_{1}^{(k)}\right)-\sigma_{1}^{(k)}
\end{array}\right) .
\end{aligned}
$$

3. Solve linear system for the increment $\delta \boldsymbol{x}^{(k)}$ :

$$
\boldsymbol{J}^{(k)} \delta \boldsymbol{x}^{(k)}=\boldsymbol{R}^{(k)}
$$

4. Update solution: $\boldsymbol{x}^{(k+1)}=\boldsymbol{x}^{(k)}-\delta \boldsymbol{x}^{(k)}$.

5. Check convergence:

$$
\begin{aligned}
& \text { IF }\|\boldsymbol{R}\|<\epsilon \text { STOP } \\
& \text { ELSE Set } k \leftarrow k+1 \text { and GOTO } 2 .
\end{aligned}
$$

Figure 27. Newton algorithm for obtaining the 1-shock curve.

\section{A.3 Rarefaction-shock curves}

Rarefaction-shock curves $\mathcal{R}_{i} \mathcal{S}_{i}\left(\boldsymbol{u}_{l}, \boldsymbol{u}_{r}\right)$ are composite waves, consisting of a rarefaction curve emanating from a left state $\boldsymbol{u}_{l}$, and a shock curve that ends at the right state $\boldsymbol{u}_{r}$. Both curves join at an intermediate state $\boldsymbol{u}_{*}$, called the post-shock state.

We now detail the procedure to compute rarefaction-shock curves of the 1-characteristic family. Assume that the left state $\boldsymbol{u}_{l}$ and the first component $u_{r}$ of the right state are known. A complete description of the 1rarefaction-shock requires: the second component $v_{r}$ of the right state, the speed $\sigma_{1}$ of the shock, and the post-shock value $\boldsymbol{u}_{*}$. These unknowns are 
R. Juanes and T. W. Patzek: Analytical solution to the Riemann problem ... 51

1. Set $k=0$. Initial guess $v_{r}^{(0)}$.

2. Predictor:

(i) Integrate along rarefaction curve $\mathcal{R}_{1}\left(\boldsymbol{u}_{l}\right)$ as in Equation (70) until

$$
\nu_{1}(\tilde{\boldsymbol{u}})=\sigma_{1}\left(\boldsymbol{u}_{r}^{(k)} ; \tilde{\boldsymbol{u}}\right)=\frac{f\left(\boldsymbol{u}_{r}^{(k)}\right)-f(\tilde{\boldsymbol{u}})}{u_{r}-\tilde{u}}
$$

(ii) Set $\boldsymbol{u}_{*}^{(k+1)}=\tilde{\boldsymbol{u}}$ at which integration terminated.

3. Corrector: Solve for $\sigma_{1}^{(k+1)}$ and $v_{r}^{(k+1)}$ by imposing that the right state belongs to the shock curve passing through the post-shock value, $\boldsymbol{u}_{r}^{(k+1)} \in \mathcal{S}_{1}\left(\boldsymbol{u}_{*}^{(k+1)}\right)$, using the Newton algorithm in Figure 27.

4. Check convergence:

$\mathrm{IF}\left|v_{r}^{(k+1)}-v_{r}^{(k)}\right|<\epsilon \mathrm{STOP}$

ELSE Set $k \leftarrow k+1$ and GOTO 2 .

Figure 28. Predictor-corrector algorithm for obtaining the rarefactionshock curve of the 1-characteristic family.

obtained using the predictor-corrector algorithm of Figure 28. A schematic of the $k$ th iteration is shown in Figure 29.

It is important to note that the post-shock value $\boldsymbol{u}_{*}$, at which the $\mathcal{R}_{1}$ and $\mathcal{S}_{1}$ curves are connected, depends on both the left and the right states. This connection is always very smooth. In fact, it can be shown [25] that both curves are connected with second order tangency (same slope and curvature). This property ensures that the predictor-corrector algorithm in Figure 28 will achieve global quadratic convergence when the initial guess is close to the solution.

The algorithm to compute a rarefaction-shock of the 2-characteristic fam$i l y$ is completely analogous. In this case, the left state $\boldsymbol{u}_{l}$ and the second component $v_{r}$ of the right state are known, and we use a predictor-corrector algorithm to compute the first component $u_{r}$ of the right state, the speed $\sigma_{2}$ of the shock, and the post-shock value $\boldsymbol{u}_{*}$. 
R. Juanes and T. W. Patzek: Analytical solution to the Riemann problem ... 52

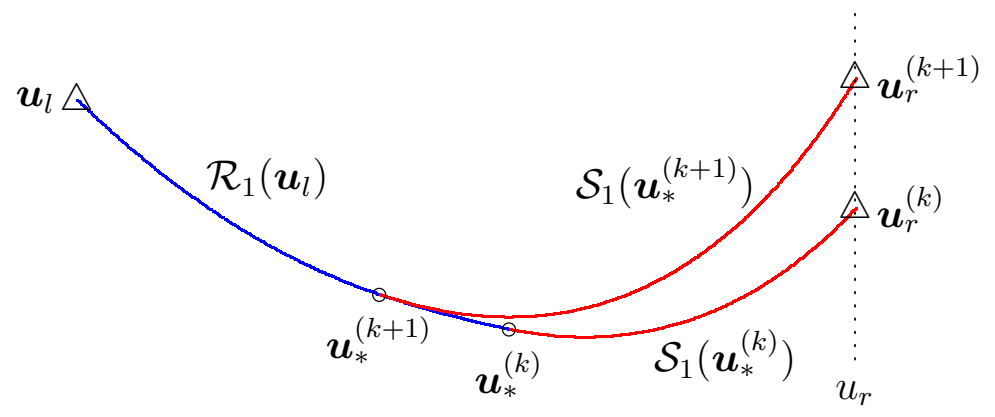

Figure 29. Schematic of the $k$ th iteration of the predictor-corrector algorithm for obtaining a rarefaction-shock of the 1-characteristic family. The rarefaction curve and the shock curve join at the post-shock value with second-order tangency. This property allows the algorithm to achieve quadratic convergence.

\section{B Solution algorithms for selected solution types of three-phase flow}

Recall that the solution of the Riemann problem of three-phase flow consists in a sequence of two waves connecting three constant states:

$$
\boldsymbol{u}_{l} \stackrel{\mathcal{W}_{1}}{\longrightarrow} \boldsymbol{u}_{m} \stackrel{\mathcal{W}_{2}}{\longrightarrow} \boldsymbol{u}_{r}
$$

The complete set of solutions (9 cases) is given in Section 4.3.2. In this appendix we describe efficient algorithms for the calculation of selected solution types. Other cases can be computed similarly.

\section{B.1 $\mathcal{S}_{1} \mathcal{S}_{2}$ solution}

The first solution we consider is that when both waves are genuine shocks, that is, $\mathcal{W}_{1} \equiv \mathcal{S}_{1}\left(\boldsymbol{u}_{l}\right)$ and $\mathcal{W}_{2} \equiv \mathcal{S}_{2}\left(\boldsymbol{u}_{r}\right)$. These two shock curves intersect at the intermediate constant state $\boldsymbol{u}_{m}$.

\section{B.1.1 Solution procedure}

The unknowns are the intermediate constant state $\boldsymbol{u}_{m}=\left(u_{m}, v_{m}\right)$ and the shock speeds $\sigma_{1}$ and $\sigma_{2}$. These 4 scalar unknowns can be found by imposing 
R. Juanes and T. W. Patzek: Analytical solution to the Riemann problem ... 53

the Rankine-Hugoniot jump condition on each shock:

$$
\begin{aligned}
f\left(u_{m}, v_{m}\right)-f\left(u_{l}, v_{l}\right) & =\sigma_{1}\left(u_{m}-u_{l}\right) \\
g\left(u_{m}, v_{m}\right)-g\left(u_{l}, v_{l}\right) & =\sigma_{1}\left(v_{m}-v_{l}\right) \\
f\left(u_{m}, v_{m}\right)-f\left(u_{r}, v_{r}\right) & =\sigma_{2}\left(u_{m}-u_{r}\right) \\
g\left(u_{m}, v_{m}\right)-g\left(u_{r}, v_{r}\right) & =\sigma_{2}\left(v_{m}-v_{r}\right)
\end{aligned}
$$

We propose using a full Newton iterative procedure to achieve quadratic convergence. The solution vector $\boldsymbol{x}$, the residual vector $\boldsymbol{R}$ and the Jacobian matrix $\boldsymbol{J}$ are given by:

$$
\begin{aligned}
\boldsymbol{x}= & \left(\begin{array}{c}
\sigma_{1} \\
v_{m} \\
u_{m} \\
\sigma_{2}
\end{array}\right) \\
\boldsymbol{R}= & \left(\begin{array}{c}
f\left(u_{m}, v_{m}\right)-f\left(u_{l}, v_{l}\right)-\sigma_{1}\left(u_{m}-u_{l}\right) \\
g\left(u_{m}, v_{m}\right)-g\left(u_{l}, v_{l}\right)-\sigma_{1}\left(v_{m}-v_{l}\right) \\
f\left(u_{m}, v_{m}\right)-f\left(u_{r}, v_{r}\right)-\sigma_{2}\left(u_{m}-u_{r}\right) \\
g\left(u_{m}, v_{m}\right)-g\left(u_{r}, v_{r}\right)-\sigma_{2}\left(v_{m}-v_{r}\right)
\end{array}\right)
\end{aligned}
$$

and

$$
\boldsymbol{J}=\left(\begin{array}{cccc}
-\left(u_{m}-u_{l}\right) & f_{, v}\left(u_{m}, v_{m}\right) & f_{, u}\left(u_{m}, v_{m}\right)-\sigma_{1} & 0 \\
-\left(v_{m}-v_{l}\right) & g_{, v}\left(u_{m}, v_{m}\right)-\sigma_{1} & g_{, u}\left(u_{m}, v_{m}\right) & 0 \\
0 & f_{, v}\left(u_{m}, v_{m}\right) & f_{, u}\left(u_{m}, v_{m}\right)-\sigma_{2} & -\left(u_{m}-u_{r}\right) \\
0 & g_{, v}\left(u_{m}, v_{m}\right)-\sigma_{2} & g_{, u}\left(u_{m}, v_{m}\right) & -\left(v_{m}-v_{r}\right)
\end{array}\right)
$$

\section{B.1.2 Admissibility of the solution}

The solution is valid if each wave satisfies the Lax entropy condition:

$$
\begin{array}{ll}
\mathcal{S}_{1}: & \nu_{1}\left(\boldsymbol{u}_{l}\right)>\sigma_{1}>\nu_{1}\left(\boldsymbol{u}_{m}\right) \\
\mathcal{S}_{2}: & \nu_{2}\left(\boldsymbol{u}_{m}\right)>\sigma_{2}>\nu_{2}\left(\boldsymbol{u}_{r}\right)
\end{array}
$$

\section{B.1.3 Example}

We show an example of a Riemann problem whose solution involves two genuine shocks. We use the relative mobilities (30)-(32), with the values 
R. Juanes and T. W. Patzek: Analytical solution to the Riemann problem ... 54

of fluid viscosities in (35). The left state is $\boldsymbol{u}_{l}=(0.25,0.2)$ and the right state is $\boldsymbol{u}_{r}=(0.15,0.8)$. Solution of the nonlinear system of algebraic equations (78) yields:

$$
\boldsymbol{u}_{m}=(0.6152,0.3278), \quad \sigma_{1}=0.1560, \quad \sigma_{2}=0.1930 .
$$

The schematic of the solution in the ternary diagram (saturation space) is depicted in Figure 30. Inflection loci (dash-dotted curves) are plotted for reference also. The intermediate constant state $\boldsymbol{u}_{m}$ is located at the intersection of the two shock curves. Note that the 1-shock is admissible even though the left and right states of the discontinuity, $\boldsymbol{u}_{l}$ and $\boldsymbol{u}_{m}$, respectively, lie on opposite sides of the 1-inflection locus. The profiles of the characteristic speeds $\nu_{1}$ and $\nu_{2}$, and the phase saturations $S_{w} \equiv u, S_{g} \equiv v$, and $S_{o} \equiv$ $1-u-v$, are plotted against the similarity variable $\zeta=x / t$ in Figure 31 .

In Figure 32 we show the evolution of the error with the number of iterations of the Newton scheme. The error at iteration $k$ is defined as:

$$
e^{(k)}:=|| \delta \boldsymbol{u}_{m}^{(k-1)} \|_{1}=\left|u_{m}^{(k)}-u_{m}^{(k-1)}\right|+\left|v_{m}^{(k)}-v_{m}^{(k-1)}\right| .
$$

The rate of convergence of the method is given by the exponent $m$ in the following expression relating the error at iteration $k+1$ with the error at iteration $k$ :

$$
e^{(k+1)} \leq C\left(e^{(k)}\right)^{m}
$$

where $C$ is a bounded positive constant. If we plot $e^{(k+1)}$ against $e^{(k)}$ in log$\log$ scale, the convergence rate is simply the asymptotic slope of the curve. As shown in Figure 32, convergence of the iterative procedure is quadratic, as expected.

\section{B.2 $\mathcal{R}_{1} \mathcal{R}_{2}$ solution}

We consider the case when both waves are rarefaction waves, so that $\mathcal{W}_{1} \equiv$ $\mathcal{R}_{1}$ and $\mathcal{W}_{2} \equiv \mathcal{R}_{2}$. There are no discontinuities, and the solution is smooth everywhere.

\section{B.2.1 Solution procedure}

The intermediate constant state is determined by the intersection of the two rarefaction curves. By contrast to the previous case, in which the intersecting curves were given by algebraic equations, rarefaction curves are defined by 
R. Juanes and T. W. Patzek: Analytical solution to the Riemann problem ... 55

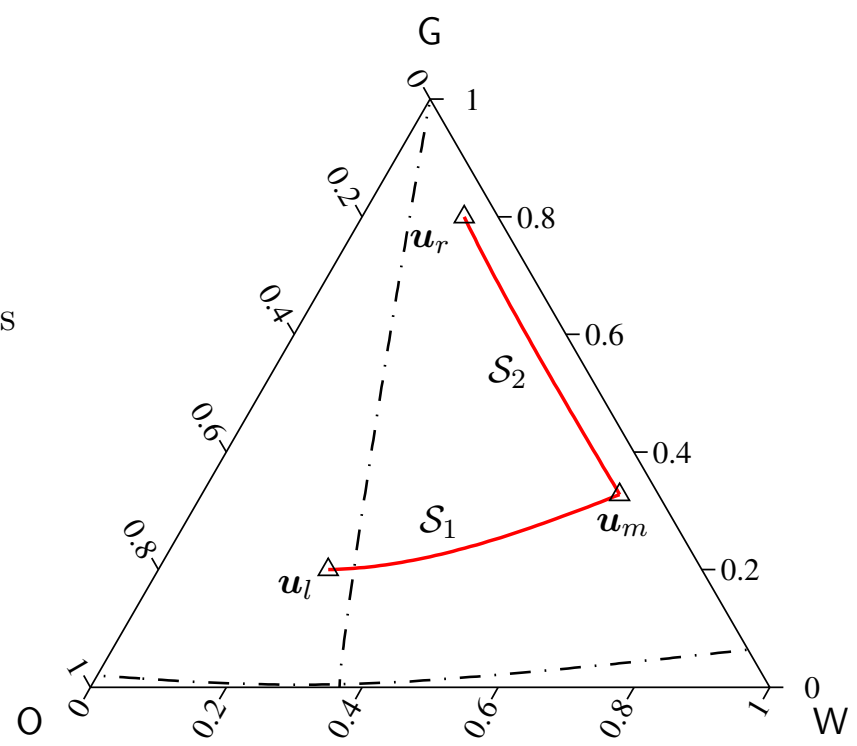

Figure 30. Schematic of the $\mathcal{S}_{1} \mathcal{S}_{2}$ solution path in the ternary diagram.
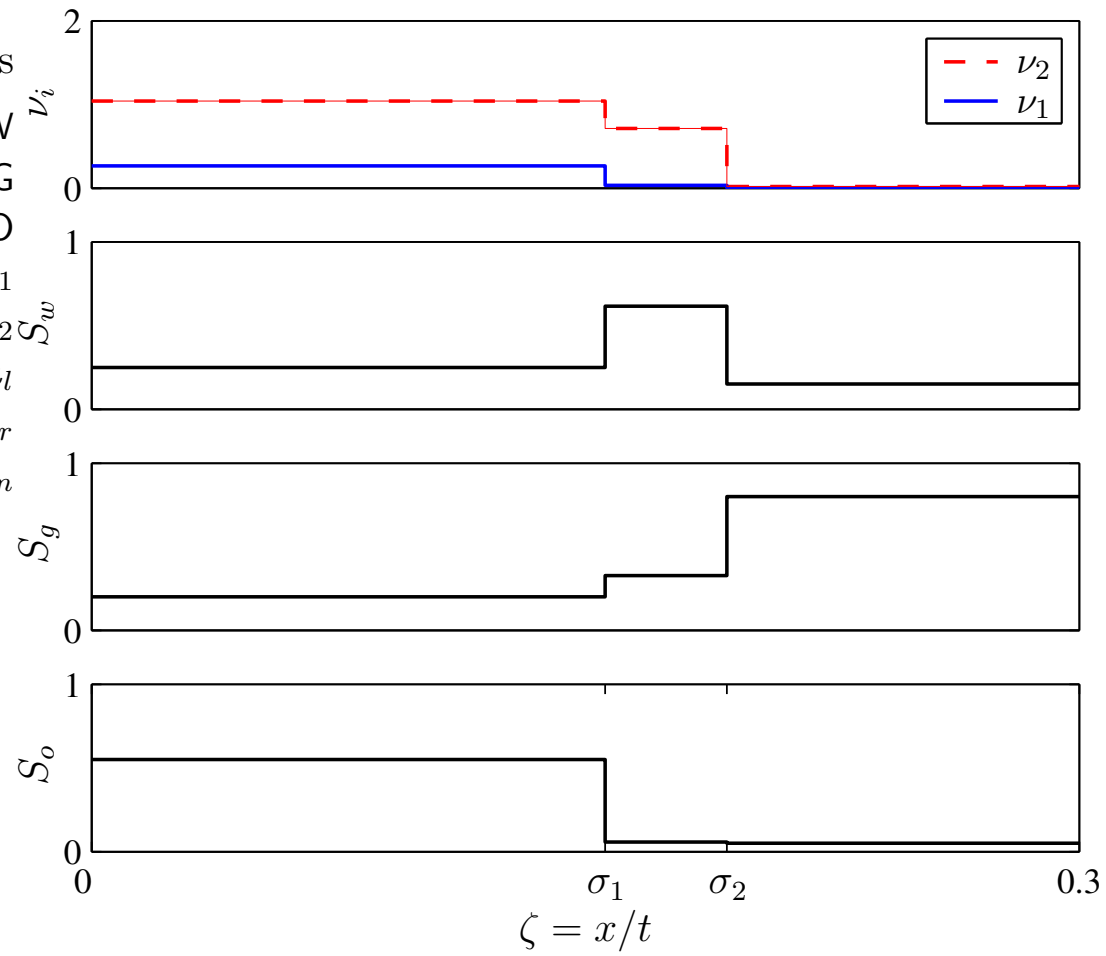

Figure 31. Profiles of wave speeds and saturations for the $\mathcal{S}_{1} \mathcal{S}_{2}$ solution. 
R. Juanes and T. W. Patzek: Analytical solution to the Riemann problem ... 56

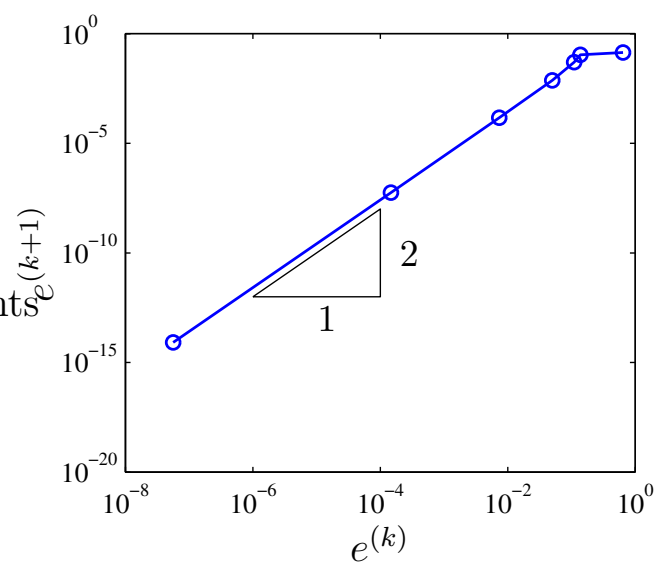

Figure 32. Performance of the Newton iterative scheme for the $\mathcal{S}_{1} \mathcal{S}_{2}$ solution, showing a quadratic rate of convergence.

differential equations (70) and (72). We suggest a predictor-corrector strategy to find $\boldsymbol{u}_{m}$ iteratively. The algorithm is given in Figure 33, and the $k$ th iteration is illustrated in Figure 34.

\section{B.2.2 Admissibility of the solution}

The solution is admissible if it is single-valued, that is:

$$
\begin{aligned}
& \mathcal{R}_{1}: \nu_{1} \text { increases monotonically along } \mathcal{R}_{1} \text { from } \boldsymbol{u}_{l} \text { to } \boldsymbol{u}_{m} \\
& \mathcal{R}_{2}: \nu_{2} \text { increases monotonically along } \mathcal{R}_{2} \text { from } \boldsymbol{u}_{m} \text { to } \boldsymbol{u}_{r}
\end{aligned}
$$

\section{B.2.3 Example}

We show an example of a Riemann problem that yields two simple rarefaction waves. We use the same relative permeability model and the same viscosities as before. The left state is $\boldsymbol{u}_{l}=(0.5,0.5)$ and the right state is $\boldsymbol{u}_{r}=(0.4,0.1)$. Solution of the $\mathcal{R}_{1} \mathcal{R}_{2}$ intersection gives the intermediate constant state:

$$
\boldsymbol{u}_{m}=(0.2658,0.3360)
$$

In Figure 35 we plot the solution in the ternary diagram. The intermediate constant state $\boldsymbol{u}_{m}$ is located at the intersection of the two rarefaction curves. Note that the saturation path never crosses the inflection 
R. Juanes and T. W. Patzek: Analytical solution to the Riemann problem ... 57

1. Set $k=0$. Initial guess $\boldsymbol{u}_{m}^{(0)}=\left(u_{m}^{(0)}, v_{m}^{(0)}\right)$.

2. Predictor:

(i) Set the integration limit $\hat{u}_{m}=u_{m}^{(k)}$ and integrate along $\mathcal{R}_{1}\left(\boldsymbol{u}_{l}\right)$ to obtain point $\hat{\boldsymbol{u}}_{m}$.

(ii) Set the integration limit $\tilde{v}_{m}=v_{m}^{(k)}$ and integrate along $\mathcal{R}_{2}\left(\boldsymbol{u}_{r}\right)$ to obtain point $\tilde{\boldsymbol{u}}_{m}$.

(iii) Compute eigenvectors $\boldsymbol{r}_{1}\left(\hat{\boldsymbol{u}}_{m}\right)$ and $\boldsymbol{r}_{2}\left(\tilde{\boldsymbol{u}}_{m}\right)$ at the integration endpoints.

3. Corrector: new approximation $\boldsymbol{u}_{m}^{(k+1)}$ is the intersection of two straight lines with orientations $\boldsymbol{r}_{1}$ and $\boldsymbol{r}_{2}$ emanating from points $\hat{\boldsymbol{u}}_{m}$ and $\tilde{\boldsymbol{u}}_{m}$, respectively.

4. Check convergence:

IF $\left\|\boldsymbol{u}_{m}^{(k+1)}-\boldsymbol{u}_{m}^{(k)}\right\|<\epsilon \mathrm{STOP}$

ELSE Set $k \leftarrow k+1$ and GOTO 2 .

Figure 33. Predictor-corrector algorithm for obtaining the $\mathcal{R}_{1} \mathcal{R}_{2}$ solution.

loci. Profiles of the wave speeds and phase saturations are shown in Figure 36. Points $a<b<c<d$ on the $x / t$-axis correspond to the wave speeds $\nu_{1}\left(\boldsymbol{u}_{l}\right)<\nu_{1}\left(\boldsymbol{u}_{m}\right)<\nu_{2}\left(\boldsymbol{u}_{m}\right)<\nu_{2}\left(\boldsymbol{u}_{r}\right)$.

Figure 37 shows the evolution of the error, defined in Equation (83), with the number of iterations of the predictor-corrector strategy. It is not surprising that convergence is quadratic, since the iterative procedure involves the eigenvectors, which are tangent to the rarefaction curves.

\section{B.3 $\mathcal{R}_{1} \mathcal{S}_{1} \mathcal{R}_{2} \mathcal{S}_{2}$ solution}

We now consider the case with the most complicated wave structure that may arise in the three-phase flow Riemann problem. In this case both waves are composite rarefaction-shocks: $\mathcal{W}_{1} \equiv \mathcal{R}_{1} \mathcal{S}_{1}$ and $\mathcal{W}_{2} \equiv \mathcal{R}_{2} \mathcal{S}_{2}$. 
R. Juanes and T. W. Patzek: Analytical solution to the Riemann problem ... 58

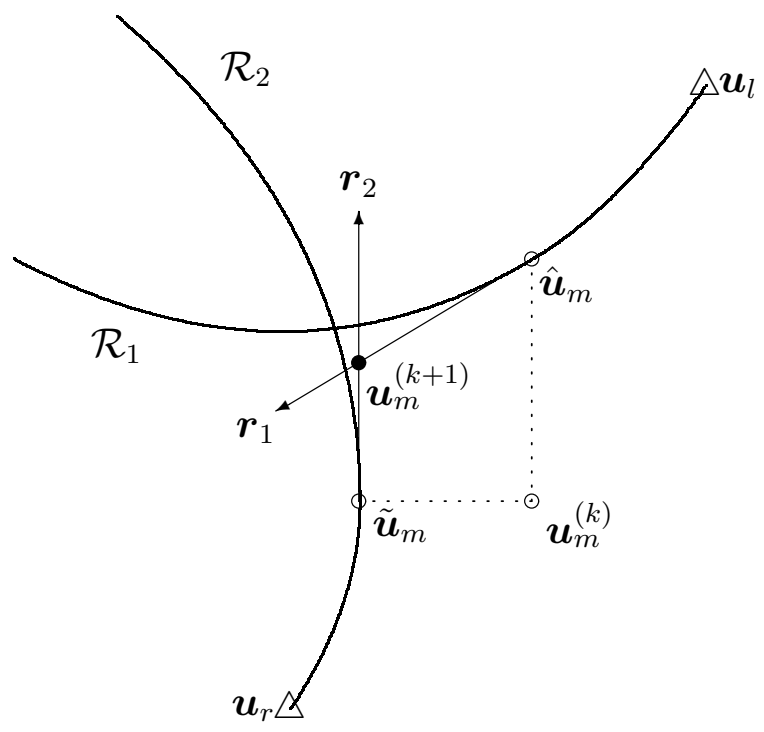

Figure 34. Schematic diagram of the $k$ th iteration of the predictorcorrector procedure for a $\mathcal{R}_{1} \mathcal{R}_{2}$ intersection.

\section{B.3.1 Solution procedure}

The variables that need to be determined to fully characterize the solution are: the intermediate constant state $\boldsymbol{u}_{m}$, the shock speeds $\sigma_{1}$ and $\sigma_{2}$, and the post-shock states $\boldsymbol{u}_{1}^{*}$ and $\boldsymbol{u}_{2}^{*}$ of each wave. The constant state $\boldsymbol{u}_{m}$ corresponds to the intersection of the two wave curves, and the post-shock states are the points where the rarefaction curve and the shock curve of the same family are joined. Schematically, this can be represented as follows:

$$
\boldsymbol{u}_{l} \stackrel{\mathcal{R}_{1}}{\longrightarrow} \boldsymbol{u}_{1}^{*} \stackrel{\mathcal{S}_{1}}{\longrightarrow} \boldsymbol{u}_{m} \stackrel{\mathcal{R}_{2}}{\longrightarrow} \boldsymbol{u}_{2}^{*} \stackrel{\mathcal{S}_{2}}{\longrightarrow} \boldsymbol{u}_{r}
$$

The major difficulty in computing the solution is that both endpoints of the $\mathcal{R}_{2}$ curve are unknown, so that the initial condition to start integration is not know a priori. The predictor-corrector algorithm in Figure 38 has proven very effective. 
R. Juanes and T. W. Patzek: Analytical solution to the Riemann problem ... 59

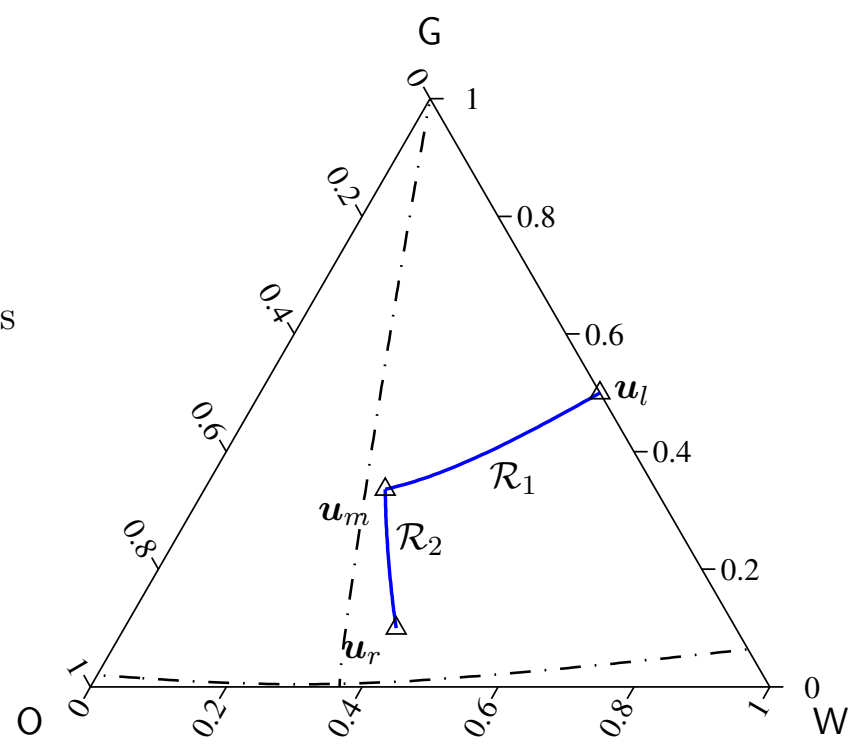

Figure 35. Schematic of the $\mathcal{R}_{1} \mathcal{R}_{2}$ solution path in the ternary diagram.
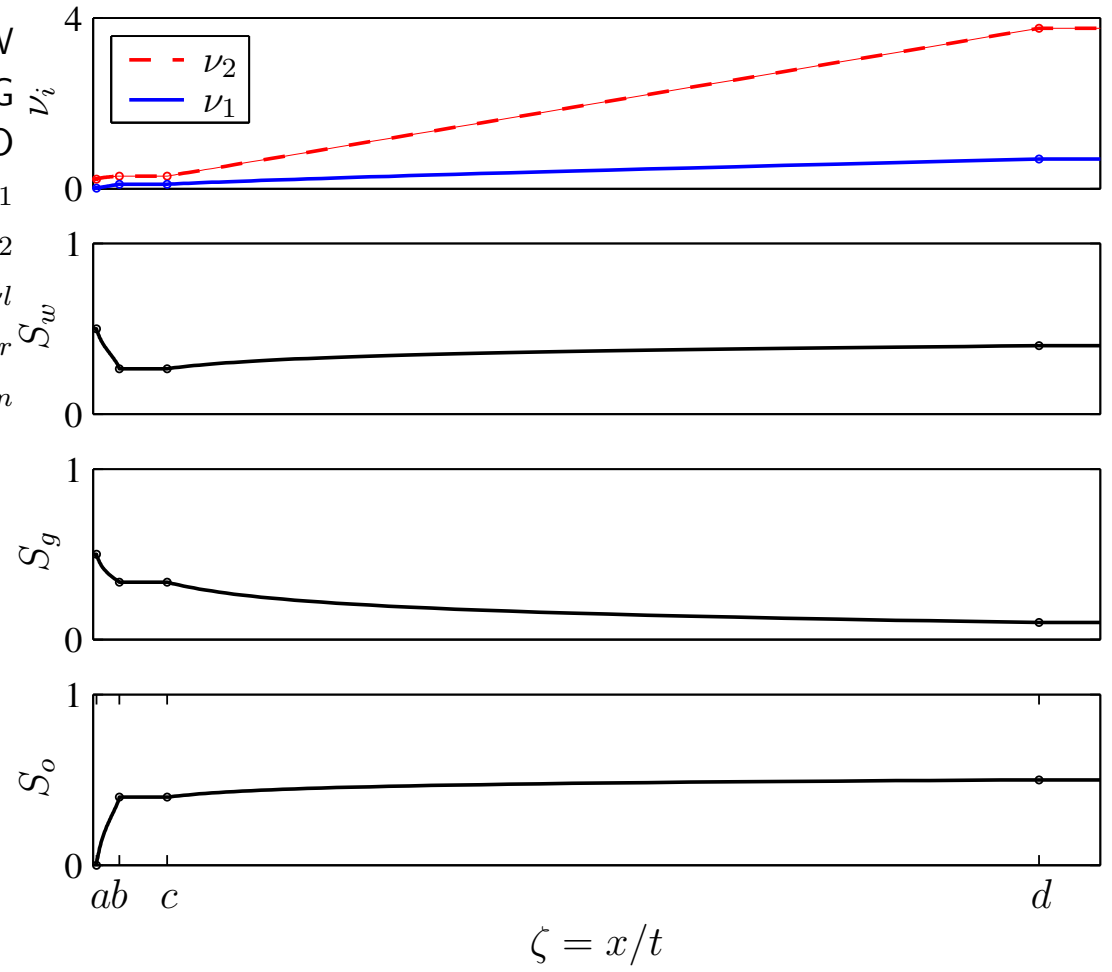

Figure 36. Profiles of wave speeds and saturations for the $\mathcal{R}_{1} \mathcal{R}_{2}$ solution. 
R. Juanes and T. W. Patzek: Analytical solution to the Riemann problem ... 60

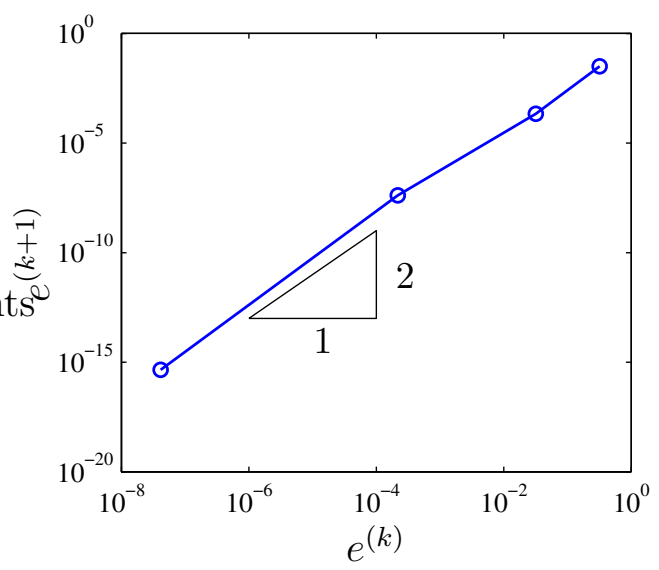

Figure 37. Performance of the predictor-corrector iterative scheme for the $\mathcal{R}_{1} \mathcal{R}_{2}$ solution, showing a quadratic rate of convergence.

1. Set $k=0$. Initial guess $\boldsymbol{u}_{m}^{(0)}=\left(u_{m}^{(0)}, v_{m}^{(0)}\right)$.

2. Predictor:

(i) Set the integration limit $\hat{u}_{m}=u_{m}^{(k)}$ and determine the $\mathcal{R}_{1} \mathcal{S}_{1}$ curve using the predictor-corrector algorithm in Figure 28, to obtain the post-shock value $\hat{\boldsymbol{u}}_{1}^{*}$ and the endpoint $\hat{\boldsymbol{u}}_{m}$.

(ii) Integrate along the $\mathcal{R}_{2}$ curve from point $\hat{\boldsymbol{u}}_{m}$ towards $\boldsymbol{u}_{r}$ until $\nu_{2}(\tilde{\boldsymbol{u}})=\sigma_{2}\left(\tilde{\boldsymbol{u}} ; \boldsymbol{u}_{r}\right)$. Set $\tilde{\boldsymbol{u}}_{2}^{*}=\tilde{\boldsymbol{u}}$ at which integration stopped.

(iii) Set $\hat{v}_{2}^{*}=\tilde{v}_{2}^{*}$ and solve the $\mathcal{S}_{2}$ curve passing through the right state $\boldsymbol{u}_{r}$ to obtain the post-shock value $\hat{\boldsymbol{u}}_{2}^{*}$.

3. Corrector: new approximation $\boldsymbol{u}_{m}^{(k+1)}$ is the intersection of curves $\mathcal{S}_{1}\left(\hat{\boldsymbol{u}}_{1}^{*}\right)$ and $\mathcal{R}_{2}\left(\hat{\boldsymbol{u}}_{2}^{*}\right)$. This is done in a similar fashion to a $\mathcal{R}_{1} \mathcal{R}_{2}$ intersection described in Section B.2.

4. Check convergence:

$\mathrm{IF}\left\|\boldsymbol{u}_{m}^{(k+1)}-\boldsymbol{u}_{m}^{(k)}\right\|<\epsilon \mathrm{STOP}$

ELSE Set $k \leftarrow k+1$ and GOTO 2 .

Figure 38. Predictor-corrector algorithm for obtaining the $\mathcal{R}_{1} \mathcal{S}_{1} \mathcal{R}_{2} \mathcal{S}_{2}$ solution. 
R. Juanes and T. W. Patzek: Analytical solution to the Riemann problem ... 61

\section{B.3.2 Admissibility of the solution}

The solution is admissible if each of the two waves is admissible individually, that is,

$$
\begin{aligned}
& \mathcal{R}_{1} \mathcal{S}_{1}:\left\{\begin{array}{l}
\nu_{1} \text { increases monotonically along } \mathcal{R}_{1} \text { from } \boldsymbol{u}_{l} \text { to } \boldsymbol{u}_{1}^{*}, \\
\nu_{1}\left(\boldsymbol{u}_{1}^{*}\right)=\sigma_{1}>\nu_{1}\left(\boldsymbol{u}_{m}\right),
\end{array}\right. \\
& \mathcal{R}_{2} \mathcal{S}_{2}:\left\{\begin{array}{l}
\nu_{2} \text { increases monotonically along } \mathcal{R}_{2} \text { from } \boldsymbol{u}_{m} \text { to } \boldsymbol{u}_{2}^{*}, \\
\nu_{2}\left(\boldsymbol{u}_{2}^{*}\right)=\sigma_{2}>\nu_{2}\left(\boldsymbol{u}_{r}\right)
\end{array}\right.
\end{aligned}
$$

\section{B.3.3 Example}

Using the same relative permeability model and the same viscosities as before, we solve the Riemann problem with left state $\boldsymbol{u}_{l}=(0.5,0.5)$ and right state $\boldsymbol{u}_{r}=(0.05,0)$. The $\mathcal{R}_{1} \mathcal{S}_{1} \mathcal{R}_{2} \mathcal{S}_{2}$ solution gives:

$$
\begin{gathered}
\boldsymbol{u}_{m}=(0.0475,0.3552), \quad \boldsymbol{u}_{1}^{*}=(0.3275,0.3618), \quad \boldsymbol{u}_{2}^{*}=(0.0499,0.0247), \\
\sigma_{1}=0.0805, \quad \sigma_{2}=7.6549
\end{gathered}
$$

In Figure 39 we represent the solution as a saturation path in the ternary diagram. It is immediate to check that the solution is admissible. Each composite wave crosses the inflection locus of the corresponding characteristic family. We note that the 2 -shock has a very small amplitude because the right state almost coincides with the inflection locus of the 2-family.

Profiles of wave speeds and phase saturations are plotted in Figure 40. We decided to split each plot into two and use a different scale on the $x / t$ axis, due to the very different speeds of the 1- and 2-wave (compare the values of $\sigma_{1}$ and $\sigma_{2}$ above). Otherwise, we would not be able to distinguish the structure of the 1-rarefaction-shock from the plots. Points $a<b<c<d$ now correspond to $\nu_{1}\left(\boldsymbol{u}_{l}\right)<\sigma_{1}<\nu_{2}\left(\boldsymbol{u}_{m}\right)<\sigma_{2}$. 
R. Juanes and T. W. Patzek: Analytical solution to the Riemann problem ... 62

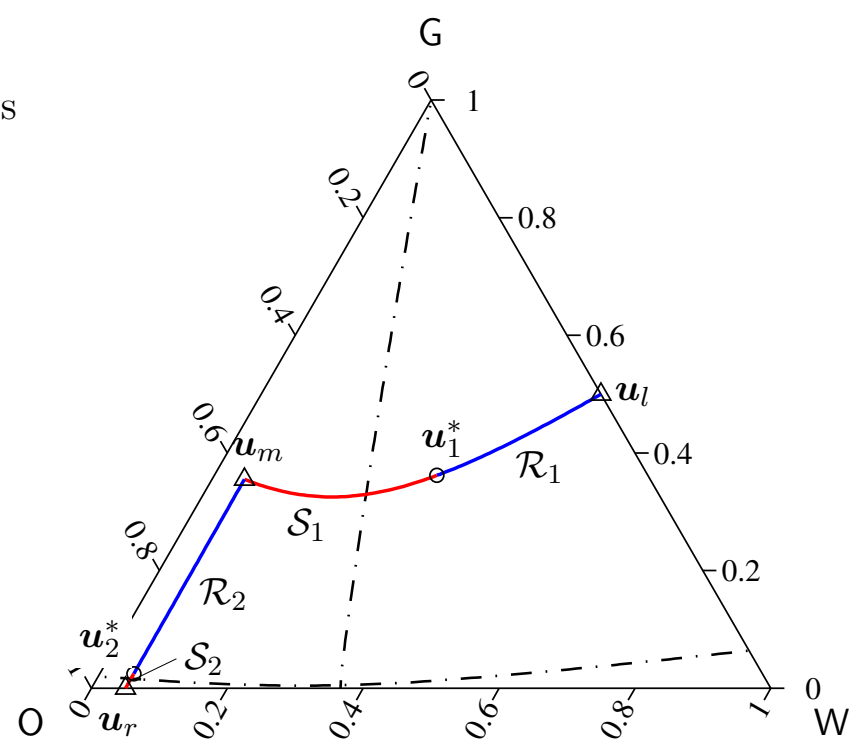

Figure 39. $\mathcal{R}_{1} \mathcal{S}_{1} \mathcal{R}_{2} \mathcal{S}_{2}$ solution path in the ternary diagram.
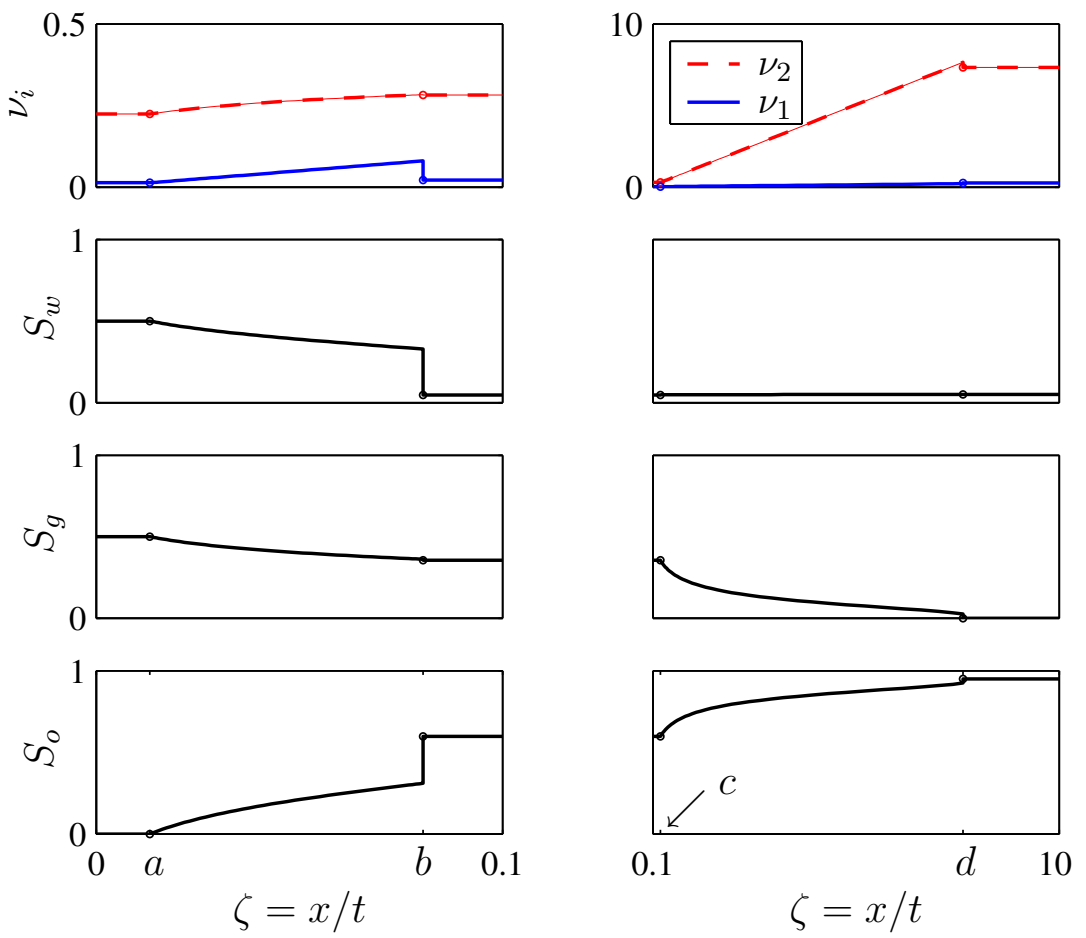

Figure 40. Wave speeds and saturations for the $\mathcal{R}_{1} \mathcal{S}_{1} \mathcal{R}_{2} \mathcal{S}_{2}$ solution. 